Meccanica

\title{
RANDOM VIBRATION MITIGATION OF BEAMS VIA TUNED MASS DAMPERS WITH SPRING INERTIA EFFECTS

\author{
--Manuscript Draft--
}

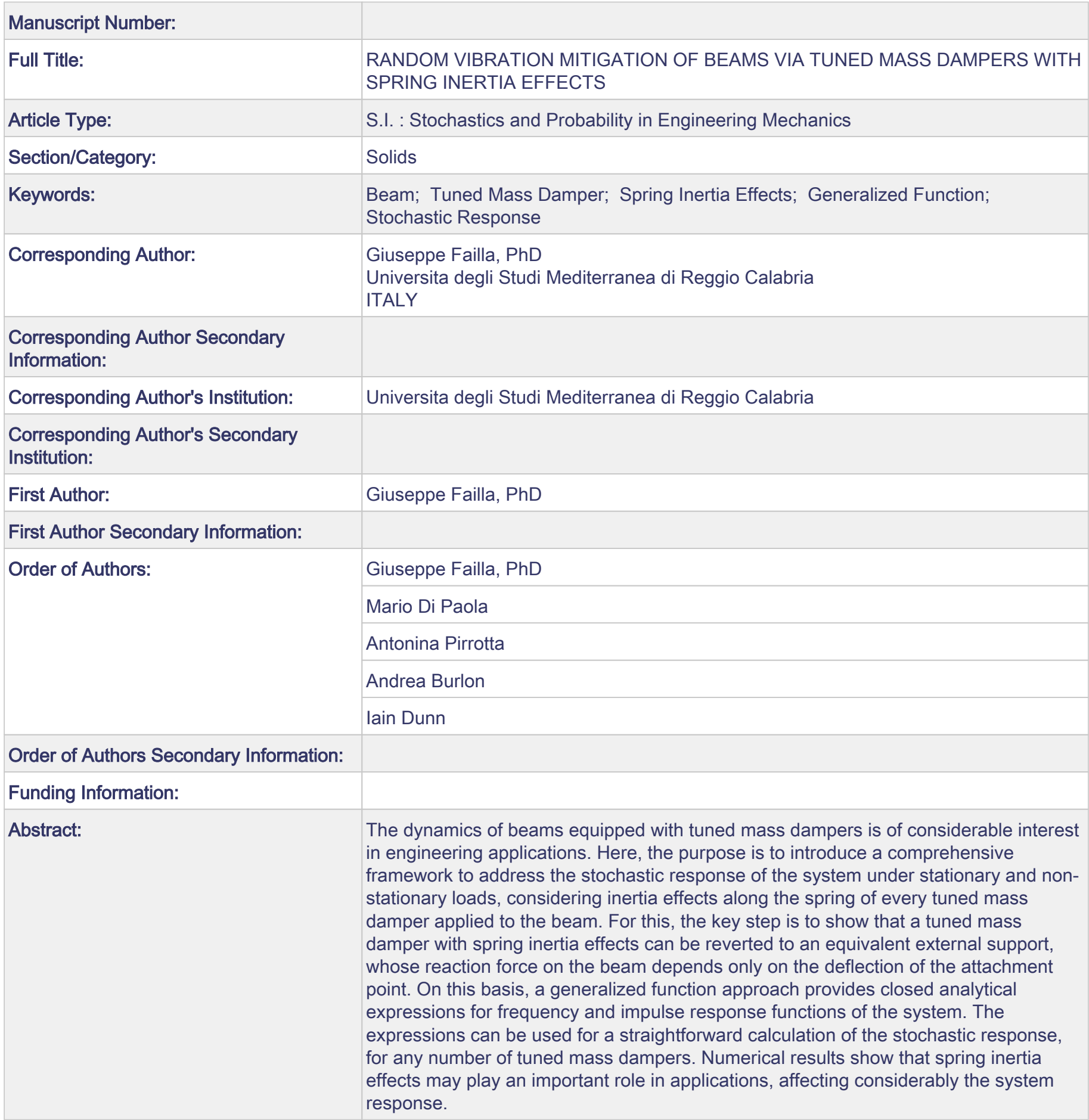




\title{
RANDOM VIBRATION MITIGATION OF BEAMS VIA TUNED MASS DAMPERS WITH SPRING INERTIA EFFECTS
}

\author{
Giuseppe Failla*, Mario Di Paola**, Antonina Pirrotta**, Andrea Burlon*, Iain Dunn** \\ *Department of Civil, Energy, Environmental and Materials Engineering (DICEAM) \\ University of Reggio Calabria, Via Graziella, 89124 Reggio Calabria, Italy \\ **Department of Civil, Environmental, Aerospace and Materials Engineering (DICAM) \\ University of Palermo, Viale delle Scienze, 90128 Palermo, Italy \\ Corresponding author: giuseppe.failla@unirc.it
}

\begin{abstract}
The dynamics of beams equipped with tuned mass dampers is of considerable interest in engineering applications. Here, the purpose is to introduce a comprehensive framework to address the stochastic response of the system under stationary and non-stationary loads, considering inertia effects along the spring of every tuned mass damper applied to the beam. For this, the key step is to show that a tuned mass damper with spring inertia effects can be reverted to an equivalent external support, whose reaction force on the beam depends only on the deflection of the attachment point. On this basis, a generalized function approach provides closed analytical expressions for frequency and impulse response functions of the system. The expressions can be used for a straightforward calculation of the stochastic response, for any number of tuned mass dampers. Numerical results show that spring inertia effects may play an important role in applications, affecting considerably the system response.
\end{abstract}

KEYWORDS: Beam, Tuned Mass Damper, Spring Inertia Effects, Generalized Function, Stochastic Response.

\section{INTRODUCTION}

Vibration mitigation via tuned mass dampers (TMDs) is a well-established approach in structural and mechanical engineering, with numerous and successful applications [1-4]. In this context, 
several studies have sought analytical or numerical solutions for beam-like structures equipped with TMDs and subjected to deterministic as well as stochastic loads [5-13].

A TMD is modelled as a lumped mass attached to the beam by a spring and, in general, a viscous dashpot in parallel. Most commonly, the inertia effects along the spring are neglected, i.e. a massless spring is considered in the model [1-13]. Yet, several studies have been devoted to include spring inertia in the analytical/numerical model of the system and investigate its effects on the performance of the TMD [14-23].

Early investigations on the role of spring inertia effects were conducted by Rayleigh [14]. He demonstrated that the fundamental frequency of a mass, attached to ground by a spring, can be approximately calculated modelling the spring as massless and adding $1 / 3$ of the spring mass to the attached mass. This approximate model, however, is rather limited because applies for a grounded system while TMDs are generally coupled with vibrating structures. Other issues are that the Rayleigh's approach calculates the fundamental frequency only and, as such, does not capture the other frequencies of the TMD associated with spring inertia. Further, inaccuracy increases as the spring mass increases compared to the attached mass, as shown by Yamamoto [15].

In view of a more accurate and general description of spring inertia effects, various studies have been proposed in the last decade. They addressed TMDs as well as mass-spring subsystems [16-23]. In general, the beam was modelled using standard Euler-Bernoulli theory, an axially-vibrating rod was used to model the spring with distributed mass; also, a dashpot in parallel with the rod was included to account for damping [18,23].

Beams coupled with mass-spring subsystems were studied in ref. [16,17]. Different methods were proposed to derive the characteristic equation: the classical method dividing the beams in consecutive segments and enforcing matching conditions between the solutions over adjacent segments [16], which provides the characteristic equation as determinant of a matrix with increasing size as the number of mass-spring subsystems increases; alternatively, the assumed-mode method, which makes use of standard free-vibration modes of the continuous beam along with a spatial linearly-varying static mode to reduce the size of the eigenvalue matrix [17]. Focusing on a cantilever beam carrying a tip mass and an in-span mass-spring subsystem, Gürgöze et al. [18] used the Hamilton's principle to derive the motion equations and obtained the characteristic equation as determinant of a $4 \times 4$ matrix via the transfer matrix method. The same system was also studied under forced vibrations, deriving the frequency response functions in exact form by coupling the steady-state equations of beam segments and rod [19]. Then, for a cantilever beam or a simplysupported beam with a mass-spring subsystem only, Gürgöze [20-21] obtained the characteristic equation in analytical form as well as the fundamental frequency via Dunkerley's procedure. Wu et 
al. [22] studied two-degree-of-freedom mass-spring subsystems attached to a beam. On demonstrating that every subsystem can be reverted to equivalent lumped masses attached to the beam, the characteristic equation was obtained by the assumed-mode method using natural frequencies and mode shapes of the bare beam. In this context, mass and stiffness matrices of the two-degree-of-freedom mass-spring subsystem were also obtained for finite element analysis.

Regarding specifically the application of TMDs, Wu [23] studied the effect of a TMD with spring inertia effects on a reference single-degree-of-freedom (SDOF) system and a beam modelled as a multi-degree-of-freedom (MDOF) system, using a standard finite element approach. The study proposed a procedure to set the optimal parameters of the TMD for the SDOF system; the same procedure was applied to the MDOF model of the beam using the orthogonality conditions for undamped modes. Numerical results were presented for the beam subjected to moving loads.

This paper presents an original approach to stochastic analysis of Euler-Bernoulli beams equipped with TMDs, where spring inertia effects are included. The key is to demonstrate that the TMD acts an external support with a pertinent equivalent stiffness. This paves the way to obtain closed analytical expressions for frequency and impulse response functions of the system, using the theory of generalized functions [24-35]. The expressions can be used to construct analytical/numerical solutions under stationary and non-stationary loads in a straightforward manner, for any number of TMDs. The solutions will provide a valuable insight into how the spring inertia effects may affect the efficiency of the TMD and more generally the dynamics of the whole system, as shown in the numerical applications.

The paper is organized as follows. The system under study is described in Section 2. The equivalent stiffness of the TMD with spring inertia effects is obtained in Section 3. Analytical expressions for frequency and impulse response functions are derived in Section 4, to finally build the stochastic response in Section 5. A numerical application is presented in Section 6. Two Appendices are included.

\section{PROBLEM STATEMENT}

Figure 1 shows a uniform Euler-Bernoulli beam coupled with an arbitrary number of TMDs, acted upon by distributed dynamic loads.

As customary, the TMD consists of a lumped mass attached to the beam by a spring and a viscous dashpot. The system will be studied under the assumption that inertia effects cannot be neglected along the spring of every TMD. That is, the spring is considered as an axially-vibrating rod with distributed mass, as indicated in Figure 1. For the beam: $L=$ length, $E I=$ flexural 
stiffness, $\rho=$ mass per unit length. For the $j^{\text {th }}$ TMD, $M_{j}=$ attached mass, $c_{j}=$ damping coefficient; $h_{j}=$ rod length,$E A_{j}=\operatorname{rod}$ axial stiffness $; \gamma_{j}=$ rod distributed mass.
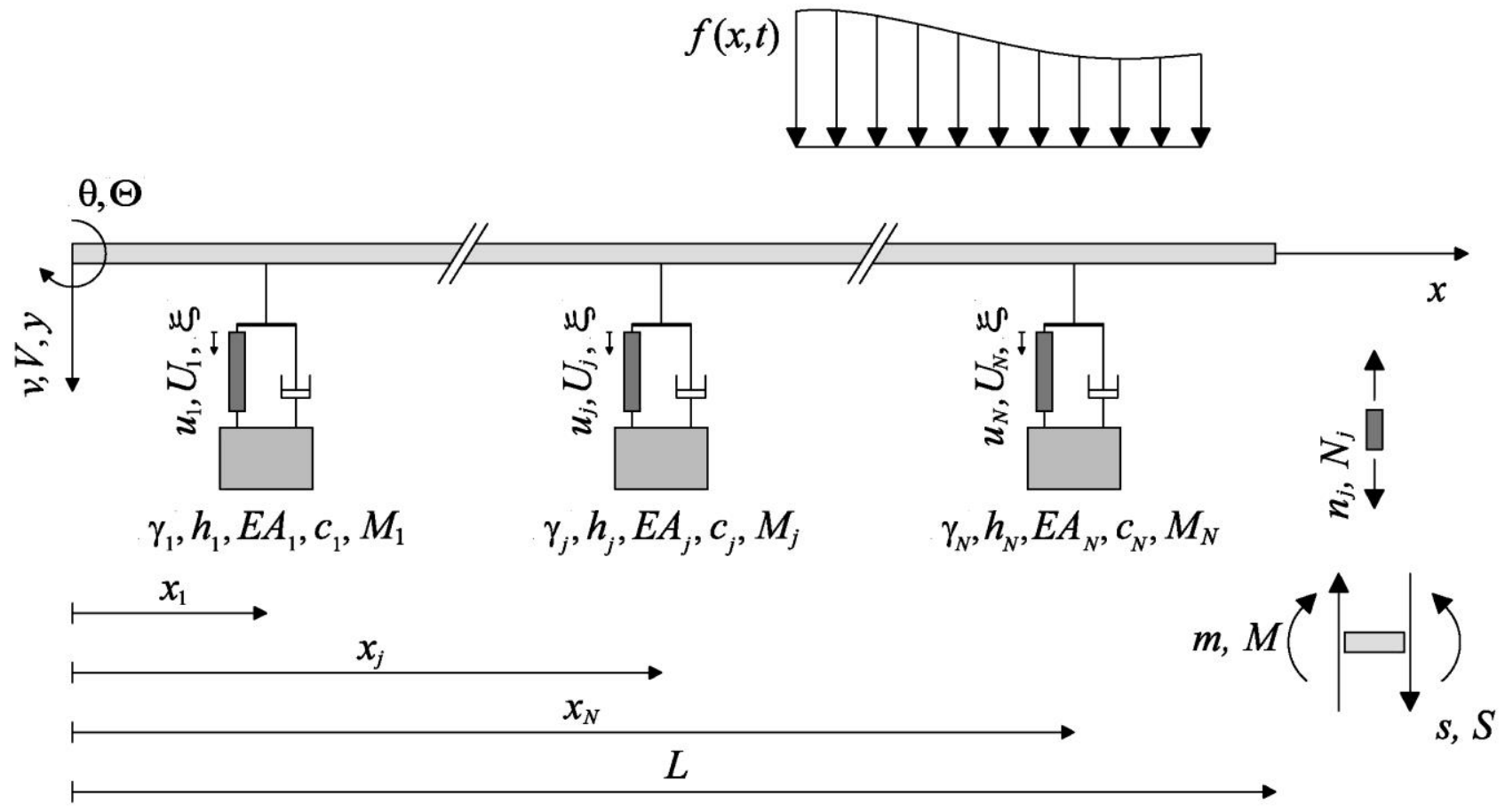

Fig. 1 Euler-Bernoulli beam coupled with TMDs where spring inertia effects are considered. Parameters and positive sign conventions are shown

\section{TUNED MASS DAMPER WITH SPRING INERTIA EFFECTS}

Assume that the vibration response of the system in Figure 1 can be represented in the form ( $\mathrm{i}=$ imaginary unit)

$$
\mathbf{y}(x, t)=\mathbf{Y e}^{\mathrm{i} \omega t} ; \quad \mathbf{z}_{j}(\xi, t)=\mathbf{Z}_{j} \mathrm{e}^{\mathrm{i} \omega t}
$$

where $\mathbf{y}=\left\{\begin{array}{lllll}v & \theta & m & s\end{array}\right\}^{T}$ and $\mathbf{Y}=\left\{\begin{array}{llll}V & \Theta & M & S\end{array}\right\}^{T}, \mathbf{z}_{j}=\left\{\begin{array}{ll}u_{j} & n_{j}\end{array}\right\}^{T}$ and $\mathbf{Z}_{j}=\left\{\begin{array}{ll}U_{j} & N_{j}\end{array}\right\}^{T}$ collect the response variables of the beam and $j^{\text {th }}$ TMD (see Figure 1 for symbols and positive sign conventions). Eqs.(1) are a general form to represent:

(a) steady-state response under an harmonic load with any frequency $\omega$, i.e. $\mathbf{Y}=\mathbf{Y}(x, \omega)$ and

$$
\mathbf{Z}_{j}=\mathbf{Z}_{j}(\xi, \omega)
$$


(b) free-vibration response, being $\omega=\omega_{n}$ an eigenvalue and $\mathbf{Y}=\mathbf{Y}_{n}(x), \mathbf{Z}_{j}=\mathbf{Z}_{j, n}(\xi)$ the corresponding vectors of eigenfunctions; in this case, eigenvalues and eigenfunctions are complex since damping in the system is not proportional.

On using Eqs.(1) in the motion equations of beam and rods, one obtains

$$
\begin{gathered}
E I \frac{\overline{\mathrm{d}}^{4} V(x)}{\mathrm{d} x^{4}}-\sum_{j=1}^{N} R_{j} \delta\left(x-x_{j}\right)-\rho \omega^{2} V(x)=0 \\
E A_{j} \frac{\mathrm{d}^{2} U(\xi)}{\mathrm{d} \xi^{2}}+\gamma_{j} \omega^{2} U(\xi)=0 \quad j=1,2, \ldots N
\end{gathered}
$$

where $\omega$-dependence is omitted for brevity. In Eq.(2), bar means generalized derivative, symbol $\delta\left(x-x_{j}\right)$ denotes a Dirac's delta at $x=x_{j}, R_{j}$ is the reaction force exerted on the beam by the $j^{\text {th }}$ TMD, i.e.

$$
R_{j}=N_{j}(0)+c_{j}(\mathrm{i} \omega)\left[U_{j}\left(h_{j}\right)-V\left(x_{j}\right)\right]
$$

being $N_{j}(0)=E A_{j} \mathrm{~d} U /\left.\mathrm{d} \xi\right|_{\xi=0}$ the axial force at $\xi=0$ in the rod.

Next, the objective is express the reaction force $R_{j}$ in Eq.(4) as a function of $V\left(x_{j}\right)$ only, which is the beam deflection at the attachment point $x=x_{j}$. For this purpose, it is seen that Eq.(3) is readily solvable in the following closed analytical form

$$
U_{j}(x)=A_{j 1} \cos \left(\alpha_{j} \omega x\right)+A_{j 2} \sin \left(\alpha_{j} \omega x\right)
$$

where $\alpha_{j}=\sqrt{\gamma_{j} / E A_{j}}$, while $A_{j 1}, A_{j 2}$ are integration constants to be set depending on the following boundary conditions (B.C.) at $\xi=0$ and $\xi=h_{j}$, respectively:

$$
\begin{gathered}
U_{j}(0)=V\left(x_{j}\right) \\
N_{j}\left(h_{j}\right)=\left.E A_{j} \frac{\mathrm{d} U}{\mathrm{~d} \xi}\right|_{\xi=h_{j}}=M_{j} \omega^{2} U\left(h_{j}\right)-c_{j}(\mathrm{i} \omega)\left[U_{j}\left(h_{j}\right)-V\left(x_{j}\right)\right]
\end{gathered}
$$


Specifically, Eq.(7) is derived from the equilibrium of the attached mass $M_{j}$. Now, Eqs.(6)-(7) yield $A_{j 1}, A_{j 2}$ in the following form

$$
\begin{gathered}
A_{j 1}=V\left(x_{j}\right) \\
A_{j 2}=V\left(x_{j}\right) \frac{\mathrm{i} c_{j}+\left(M_{j} \omega-\mathrm{i} c_{j}\right) \cos \left(\alpha_{j} \omega h_{j}\right)+\beta_{j} \sin \left(\alpha_{j} \omega h_{j}\right)}{\beta_{j} \cos \left(\alpha_{j} \omega h_{j}\right)-\left(M_{j} \omega-\mathrm{i} c_{j}\right) \sin \left(\alpha_{j} \omega h_{j}\right)}
\end{gathered}
$$

where $\beta_{j}=\sqrt{\gamma_{j} E A_{j}}$, and Eqs.(8) can be used in Eq.(5) for $U_{j}(x)$ to obtain $R_{j}$ in Eq.(4) as:

$$
R_{j}=-\kappa_{j}(\omega) V\left(x_{j}\right)
$$

where

$$
\kappa_{j}(\omega)=\frac{f_{j}(\omega)}{w_{j}(\omega)}-\mathrm{i} c_{j} \omega\left[\cos \left(\alpha_{j} \omega h_{j}\right)-\sin \left(\alpha_{j} \omega h_{j}\right) \frac{f_{j}(\omega)}{w_{j}(\omega)}-1\right]
$$

with

$$
\begin{gathered}
f_{j}(\omega)=\beta_{j} \omega\left\{-c_{j}+c_{j} \cos \left(\alpha_{j} \omega h_{j}\right)+\mathrm{i}\left[M_{j} \omega \cos \left(\alpha_{j} \omega h_{j}\right)+\beta_{j} \sin \left(\alpha_{j} \omega h_{j}\right)\right]\right\} \\
w_{j}(\omega)=c_{j} \sin \left(\alpha_{j} \omega h_{j}\right)+\mathrm{i}\left[M_{j} \omega \sin \left(\alpha_{j} \omega h_{j}\right)-\beta_{j} \cos \left(\alpha_{j} \omega h_{j}\right)\right]
\end{gathered}
$$

Eq.(9) is the sought form of the reaction force $R_{j}$ as a function of $V\left(x_{j}\right)$ only, i.e. the beam deflection at the attachment point $x=x_{j}$.

For comparison, recognize that Eq.(10) for $\kappa_{j}(\omega)$ reverts to existing results for specific parameters. For instance,

$$
\lim _{\gamma_{j} \rightarrow 0} \kappa_{j}(\omega)=\frac{M_{j} \omega^{2}\left(E A_{j} / h_{j}+\mathrm{i} c_{j} \omega\right)}{\left(M_{j} \omega^{2}-\mathrm{i} c_{j} \omega\right)-E A_{j} / h_{j}}
$$


which holds for a TMD where spring inertia effects are neglected, being $k_{j}=E A_{j} / h_{j}$ the stiffness of the massless spring [9]. On the other hand, for $c_{j}=0$ Eq.(13) matches Eq.(30) in ref. [36], which holds for an undamped mass $M_{j}$ attached to the beam by a spring of stiffness $k_{j}=E A_{j} / h_{j}$.

\section{ANALYTICAL SOLUTIONS IN FREQUENCY AND TIME}

Eq.(9) is the key point of this study: providing indeed the reaction force as a function of the deflection $V\left(x_{j}\right)$, Eq.(9) will allow to calculate the response of the whole system using the motion equation of the beam only, i.e. without considering the motion equations of the TMDs. The calculation will be pursued generalizing the approach recently proposed by some of the authors in ref. $[9,30,32]$, in both frequency and time domains. Remarkably, the approach will provide the responses along the beam and in every TMD, as explained in the following.

\subsection{Exact frequency response}

Be the beam in Figure 1 subjected to harmonic loads of frequency $\omega$, so that the steady-state response variables take the form (1). Based on ref. [30], it can be written that

$$
\mathbf{Y}(x, \omega)=\mathbf{W}(x, \omega) \mathbf{d}+\mathbf{Y}^{(f)}(x, \omega)
$$

where $\mathbf{d}=\left\{\begin{array}{llll}d_{1} & d_{2} & d_{3} & d_{4}\end{array}\right\}^{T}$ is a vector of integration constants, $\mathbf{W}(x, \omega)$ is a $4 \times 4$ matrix depending on the solution to the homogeneous equation associated with Eq.(2), while $\mathbf{Y}^{(f)}(x, \omega)$ is a $4 \times 1$ load-dependent vector associated with particular integrals of Eq.(2); specifically, the reaction force $R_{j}$ used in Eq.(2) is given as Eq.(9). Every element in matrix $\mathbf{W}$ and vector $\mathbf{Y}^{(f)}$ is available in an exact closed analytical form, as summarized in Appendix A.

The beam B.C. shall be enforced to obtain $\mathbf{d}$, deriving the following set of equations

$$
\mathbf{B d}=\mathbf{r} \rightarrow \mathbf{d}=\mathbf{B}^{-1} \mathbf{r}
$$

where $\mathbf{B}$ and $\mathbf{r}$ are a $4 \times 4$ matrix and a $4 \times 1$ vector, built from $\mathbf{W}$ and $\mathbf{Y}^{(f)}$ computed at the beam ends, $x=0$ and $x=L$. 
The inverse matrix $\mathbf{B}^{-1}$ is available in a closed analytical form, as shown in ref. [30]. Hence, replacing Eq.(15) for $\mathbf{d}$ in Eq.(14) leads to a closed analytical expression for the frequency response $\mathbf{Y}(x, \omega)$ of the beam in Figure 1 .

It is noteworthy that Eq.(14) for $\mathbf{Y}(x, \omega)$ is an exact closed analytical expression, which holds for any number of TMDs along the beam. The frequency response $\mathbf{Z}_{j}(\xi, \omega)$ in every TMD can be obtained from the deflection $V\left(x_{j}, \omega\right)$ of the attachment point, using Eq.(5) with $A_{j 1}, A_{j 2}$ given by Eqs.(8). Obviously, $U_{j}\left(h_{j}, \omega\right)$ provides the displacement of the mass $M_{j}$. Pertinent expressions are Eqs.(A.9) in Appendix A.

Eq.(15) holds for homogeneous B.C.; non-homogeneous B.C. can still be considered as homogeneous, with an end TMD modelled as internal damper located at $x=0^{+}$and $x=L^{-}$.

\subsection{Modal response functions}

Next, be the beam in Figure 1 subjected to an impulsive loading $p(x, t)=p(x) \delta(t)$, where $\delta(t)$ denotes a Dirac's delta in time and $p(x)$ is a space-dependent function. Following the approach in ref. [9,32], the vector of impulse response functions $\mathbf{h}=\left\{\begin{array}{llll}h_{v} & h_{\theta} & h_{m} & h_{s}\end{array}\right\}^{T}$ of the beam can be represented by modal superposition as

$$
\mathbf{h}(x, t)=\sum_{k=1}^{\infty} \mathbf{h}_{k}(x, t)=\sum_{k=1}^{\infty} g_{k}(t) \mathbf{Y}_{k}(x)
$$

where, for the impulsive nature of the loading, $g_{k}(t)$ is given the form $[37,38]$

$$
g_{k}(t)=\tilde{g}_{k} \mathrm{e}^{\mathrm{i} \omega_{k} t}
$$

being $\tilde{g}_{k}$ a complex coefficient, while $\omega_{k}$ and $\mathbf{Y}_{k}(x)$ are eigenvalue and vector of eigenfunctions associated with the $k^{\text {th }}$ mode. Namely, $\omega_{k}$ and $\mathbf{Y}_{k}(x)$ are complex, being damping not proportional. The eigenvalue problem is $\mathbf{B}(\omega) \mathbf{d}=\mathbf{0}$, i.e. Eq.(15) with $\mathbf{r}=\mathbf{0}$.

The approach in ref. $[9,32]$ can be applied to obtain the following closed analytical expression of the coefficient $\tilde{g}_{k}$, 
where $\eta_{k}$ is the projection of the space-dependent loading function $p(x)$ on the eigenfunction $V_{k}(x)$

$$
\eta_{k}=\int_{0}^{L} p(x) V_{k}(x) \mathrm{d} x
$$

and

$$
\Pi_{k}=\sum_{j=1}^{N} \sum_{l=1}^{4} \frac{r_{j, l}\left(\omega_{k}\right)}{s_{j}\left(\omega_{k}\right)} V_{k}^{2}\left(x_{j}\right)+2 \rho \int_{0}^{L} V_{k}^{2}(x) d x
$$

where $r_{j, l}\left(\omega_{k}\right)$ are functions given in Appendix B, for brevity. For the derivation of Eq.(18), the key is the analytical expression (10) derived for the eigenvalue-dependent stiffness $\kappa_{j}(\omega)$ as explained, again, in Appendix B.

Now, for damping levels generally encountered in vibration engineering applications, it is known that modes contributing to the beam response occur in complex conjugate pairs, i.e. $g_{k}(t)$ in Eq.(16) is $g_{k}(t)=\tilde{g}_{k} \mathrm{e}^{\mathrm{i} \omega_{k} t}$ as well as $g_{k}(t)=\tilde{g}_{k}^{*} \mathrm{e}^{-\mathrm{i} \omega_{k} t}$, with $\tilde{g}_{k}^{*}$ complex conjugate of $\tilde{g}_{k}$. The result is the following real form of the modal impulse response function for the $k^{\mathrm{h}}$ mode in Eq.(16):

$$
\mathbf{h}_{k}(x, t)=\boldsymbol{\varphi}_{k}(x)\left|\omega_{k}\right| z_{k}(t)+\boldsymbol{\psi}_{k}(x) \dot{z}_{k}(t)
$$

where

$$
\begin{aligned}
& \boldsymbol{\varphi}_{k}(x)=\zeta_{k} \boldsymbol{\Psi}_{k}(x)-\sqrt{1-\zeta_{k}^{2}} \lambda_{k}(x) \\
& \boldsymbol{\Psi}_{k}(x)=2 \operatorname{Re}\left[\tilde{g}_{k} \mathbf{Y}_{k}(x)\right] ; \quad \boldsymbol{\lambda}_{k}(x)=2 \operatorname{Im}\left[\tilde{g}_{k} \mathbf{Y}_{k}(x)\right] \\
& z_{k}(t)=\frac{1}{\omega_{D k}} e^{-\zeta_{k}\left|\omega_{k}\right| t} \sin \left(\omega_{D k} t\right) \quad \omega_{D k}=\left|\omega_{k}\right| \sqrt{1-\zeta_{k}^{2}}
\end{aligned}
$$


Finally, based on ref. $[9,32]$ the following expression may be derived for the modal frequency

response function $\mathbf{H}_{k}=\left\{\begin{array}{llll}H_{v, k} & H_{\theta, k} & H_{m, k} & H_{s, k}\end{array}\right\}^{T}$

$$
\mathbf{H}_{k}(x, \omega)=\boldsymbol{\varphi}_{k}(x)\left|\omega_{k}\right| H_{k}(\omega)+\boldsymbol{\psi}_{k}(x) \mathrm{i} \omega H_{k}(\omega)
$$

where

$$
H_{k}(\omega)=\frac{1}{\left|\omega_{k}\right|^{2}-\omega^{2}+\mathrm{i} 2 \zeta_{k}\left|\omega_{k}\right| \omega}
$$

Eq.(25) complements the exact frequency response (14) as provides an insight into the contribution of individual modes, i.e.

$$
\mathbf{Y}(x, \omega) \approx \sum_{k=1}^{n} \mathbf{H}_{k}(x, \omega)
$$

where $n$ is the number of modes retained in the modal representation of the response.

Expressions for the impulse and frequency response functions in every TMD readily follows from Eq.(16) and Eq.(27) provided that $\mathbf{Y}_{k}(x)$ is replaced with $\mathbf{Z}_{j, k}(x)$, i.e. the vector of eigenfunctions associated with the $k^{\text {th }}$ mode for axial displacement and axial force in the $j^{\text {th }}$ TMD. Specifically, $\mathbf{Z}_{j, k}(x)$ is calculated from the deflection $V\left(x_{j}\right)$ of the attachment point, using Eqs.(A.9) in Appendix A.

It is noteworthy that Eq.(16) and Eq.(27) for the impulse and frequency response functions hold for any number of TMDs along the beam. Every modal contribution (21) and (25) is exact and readily obtainable in closed analytical form, once the eigenvalues are calculated.

Finally, notice that Eq.(16) and Eq.(27) hold also for TMDs where spring inertia effects are neglected. Indeed, the limit of Eq.(20) for vanishing rod mass $\gamma_{j} \rightarrow 0$ provides the following term:

$$
\lim _{\gamma_{j} \rightarrow 0} \Pi_{k}=\sum_{j=1}^{N} \frac{M_{j}\left[2\left(E A_{j} / h_{j}+\mathrm{i} \omega_{k} c_{j}\right)^{2}-\mathrm{i} \omega_{k}^{3} M_{j} c_{j}\right]}{\left[\left(E A_{j} / h_{j}+\mathrm{i} \omega_{k} c_{j}\right)-M_{j} \omega_{k}^{2}\right]^{2}} V_{k}^{2}\left(x_{j}\right)+2 \rho \int_{0}^{L} V_{k}^{2}(x) d x
$$

which was already derived for standard TMDs without spring inertia effects in ref. [9]. 


\section{STOCHASTIC RESPONSE}

Now, the stochastic response of the beam in Figure 1 can be computed taking advantage of the equations derived in Sections 3-4.

The power spectral density (PSD) matrix of the stationary response to an arbitrary load $p(x) f(t)$, being $f(t)$ a process with PSD denoted by $S_{f f}(\omega)$, can readily be obtained as

$$
\mathbf{S}_{\mathbf{Y Y}}(x, \omega)=\mathbf{Y}(x, \omega)\left(\mathbf{Y}^{*}(x, \omega)\right)^{T} S_{f f}(\omega)
$$

where the frequency response vector $\mathbf{Y}(x, \omega)$ can be obtained by two approaches: (a) the exact approach in Section 4.1, see Eq.(14); (b) the approximate modal approach in Section 4.2, see Eq.(27). Both are analytical thanks to the closed form expressions derived in Section 4.

The response to non-stationary loads can be obtained by an efficient Monte Carlo simulation which relies on the closed analytical expressions for the impulse response functions (16). That is, every sample of the response process can be built by the Duhamel convolution integral:

$$
\mathbf{y}(x, t)=\int_{0}^{t} \mathbf{h}(x, t-\tau) f(\tau) d \tau
$$

Namely, numerical or analytical solutions are obtainable from Eq.(30); analytical solutions are typical of exponentially-modulated harmonic loads, as shown in ref. [9].

Eq.(29) and Eq.(30) provide the response variables along the beam. The same equations can be used to calculate the response in every TMD, using the pertinent frequency response functions as well as the eigenfunctions in the TMD. Indeed, the latter can be derived from the deflection of the attachment point using Eqs.(A.9) in Appendix A.

It is noteworthy that the analytical solutions built by Eq.(29), as well as the analytical/numerical solutions built by Eq.(30), involve remarkable advantages compared to a standard FE approach. Indeed, the latter provides only numerical solutions with accuracy depending on mesh refinement and requires updating/refining the mesh whenever TMDs and loads change position along the beam.

\section{NUMERICAL APPLICATION}

Consider the cantilever beam in Figure 2, equipped with a TMD at the tip. Beam parameters are: $L=6 \mathrm{~m}, E I=45 \times 10^{8} \mathrm{Nm}^{2}, \quad=156.25 \mathrm{kgm}^{1}$. For the TMD: the lumped mass is approximately 
$1 \%$ of the beam mass, i.e. $M_{1}=9.375 \mathrm{~kg}$; the rod distributed mass $\quad{ }_{1}$ is taken such that $\gamma_{1} h_{1}=10 \%$ of the lumped mass $M_{1}$; various values for the damping coefficient $c_{1}$ will be selected for comparison. Axial stiffness $E A_{1}$ and length $h_{1}$ of the rod are calibrated so that the TMD is tuned to a desired natural frequency of the undamped beam. Specifically, here two tuning frequencies are considered, $3.88 \mathrm{~Hz}$ and $133.69 \mathrm{~Hz}$, which correspond to 1st and 4th natural frequency of the undamped beam. For $3.88 \mathrm{~Hz}: E A_{1}=4.8 \times 10^{3} \mathrm{~N}$ and $h_{1}=0.86 \mathrm{~m}$; while for $133.69 \mathrm{~Hz}$, $E A_{1}=5.25 \times 10^{6} \mathrm{~N}$ and $h_{1}=0.79 \mathrm{~m}$. The proposed method is implemented in Mathematica [39].

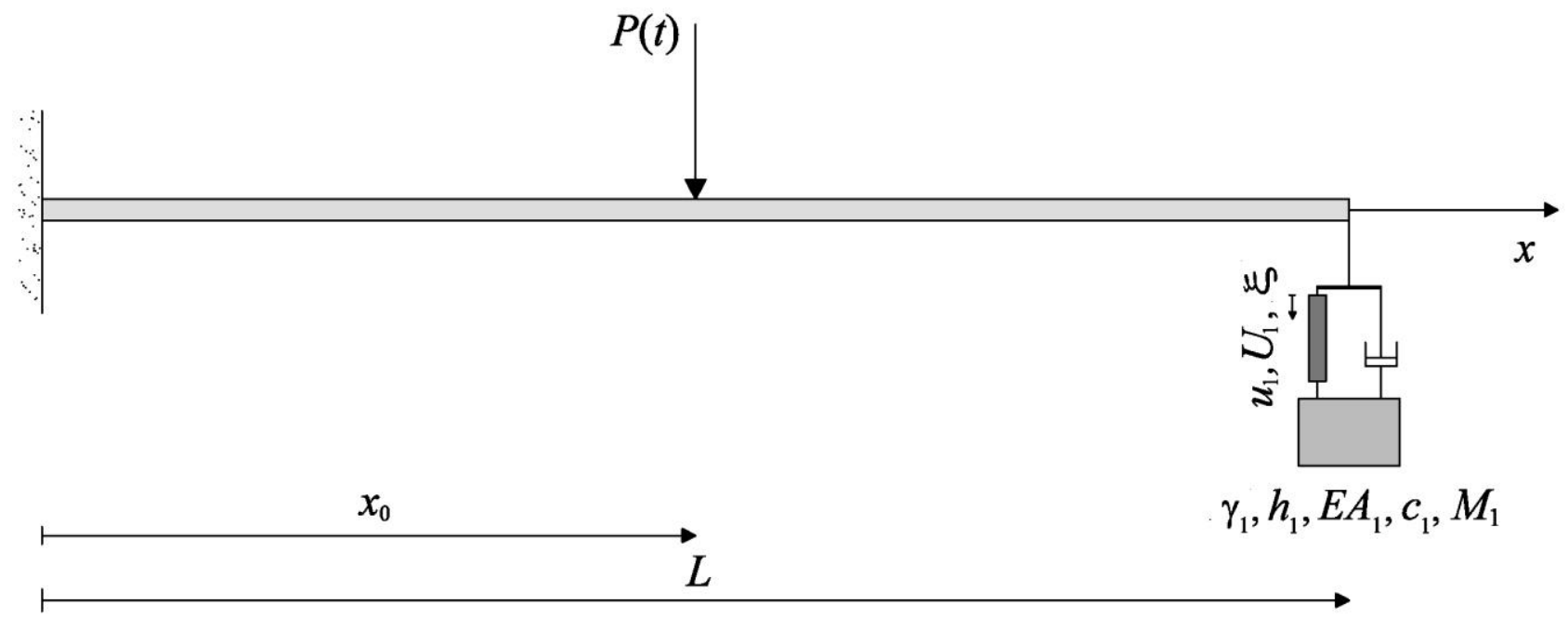

Fig. 2 Cantilever beam with a tip TMD where spring inertia effects are considered

The first step is to compare the exact frequency response (14) with the corresponding approximate one (27), obtained by complex modal superposition. For the tuning frequency $=4$ th natural frequency and damping coefficient $c_{1}=1000 \mathrm{Nm}^{-1} \mathrm{~s}$, Figure 3 shows the frequency response amplitude for the beam deflection at the tip under a concentrated harmonic load $f(x, t)=\delta\left(x-x_{0}\right) P(t)=\delta\left(x-x_{0}\right) \mathrm{e}^{\mathrm{i} \omega t}$ applied at midspan $\left(x_{0}=0.5 L\right)$, as obtained by Eq.(14) and Eq.(27) with $n=15$ modes. The two solutions are in perfect agreement. The individual contribution of every mode to the total frequency response function is evident in Figure 3, providing valuable insight into the dynamics of the system.

Next, consider the beam acted upon by a concentrated load $f(x, t)=\delta\left(x-x_{0}\right) P(t)$ applied at midspan $\left(x_{0}=0.5 L\right)$, being $P(t)$ a white-noise stationary process of one-sided PSD 
$S_{f f}(\omega)=10^{7} \mathrm{~N}^{2} \mathrm{~s}$. The exact PSD of the beam response can be computed in closed analytical form by Eq.(29), with Eq.(14) for the frequency response. Also, since the interest is to assess whether spring inertia effects play a significant role, Eq.(14) is applied using for the equivalent stiffness $\kappa_{j}(\omega)$ in Eq.(9): (a) Eq.(10) to consider spring inertia effects; (b) Eq.(13) to neglect spring inertia effects.

Figures 4-5, Figures 6-7 and Figures 8-9 report, for the two tuning frequencies of the TMD respectively, the one-sided PSD (29) of the beam deflection at the tip for three different values of the damping coefficient $c_{1}: c_{1}=10 \mathrm{Nm}^{-1} \mathrm{~s}$ (Figures 4-5), $c_{1}=100 \mathrm{Nm}^{-1} \mathrm{~s}$ (Figures 6-7), $c_{1}=1000 \mathrm{Nm}^{-1} \mathrm{~s}$ (Figures 8-9). It is apparent that, while no differences can be appreciated when the tuning frequency $=1$ st natural frequency of the undamped beam, discrepancies become considerable whereas the tuning frequency $=4$ th natural frequency of the undamped beam; it is noteworthy that this happens regardless of the amount of damping. On the other hand, for the case tuning frequency $=1$ st natural frequency, several additional peaks do appear in the PSD of the beam deflection, which are associated with the dynamics of the TMD with spring inertia effects. This means that spring inertia effects may not play a significant role in the frequency range close to the 1st natural frequency but, still, affect the beam response at higher frequencies.

For a further insight, Figures 10-11, Figures 12-13 and Figures 14-15 show the one-sided PSD (29) for the displacement of the lumped mass $M_{1}$ of the TMD, computed with and without spring inertia effects from Eqs.(A.9). From Figures 11-13-15 it is well apparent that, when the tuning frequency $=4$ th natural frequency of the undamped beam, the dynamics of the mass $M_{1}$ around the tuning frequency is affected by spring inertia effects. On the other hand, Figures 10-12-14 show that, when the tuning frequency $=1$ st natural frequency, the dynamics of the mass $M_{1}$ is not affected by spring inertia effects around the tuning frequency, but additional peaks appear at higher frequencies. As expected, these peaks match those in the PSD for the beam deflection at the tip, shown in Figures 4-6-8.

Finally, for tuning frequency $=4$ th natural frequency and damping coefficient $c_{1}=10 \mathrm{Nm}^{-1} \mathrm{~s}$, it is of interest to investigate the variance of the beam response to the load $f(x, t)=\delta\left(x-x_{0}\right) P(t)$ applied at midspan $\left(x_{0}=0.5 L\right)$, when $P(t)$ is a coloured stationary process with one-sided PSD $S_{f f}(\omega)=10^{7} \mathrm{~N}^{2} \mathrm{~s}$ defined within the following ranges: (i) $120<<135$, (ii) $145<<160$. Specifically, the ranges are centred on peaks of the response in Figure 5 around the tuning frequency $=4$ th natural frequency. The time-dependent variance of the response is calculated with 
5000 samples, with each sample built from Eq.(30). The process $P(t)$ is simulated by harmonic superposition as in ref.[40] and, in this case, closed analytical solutions may readily be found for every sample given by Eq.(30). For comparison at stationarity, the variance of the response is calculated integrating the exact PSD (29), with Eq.(14) for the frequency response. Once again, the purpose is to investigate the role of spring inertia effects. For this, Eq.(30) and Eq.(29) are used considering either Eq.(10) or Eq.(13) for the equivalent stiffness $\kappa_{j}(\omega)$ in Eq.(9), either Eq.(20) or Eq.(28) for the coefficient $\Pi_{k}$ in Eq.(18).

Figures 16-17 show the variance of the beam deflection at the tip obtained for the case (i) and (ii) respectively, considering or neglecting spring inertia effects. The first observation is that, once stationarity is attained, the variance obtained from the Monte Carlo simulations matches very well that computed via integration of the exact PSD (29). On the other hand, the most relevant comment is that ignoring spring inertia effects may cause errors in the estimation of the variance up to about $40 \%$, which may be either conservative or non-conservative depending on the input frequency range. This is an important conclusion for design purposes, especially for mechanical applications where high-frequency TMDs are frequently used [41].

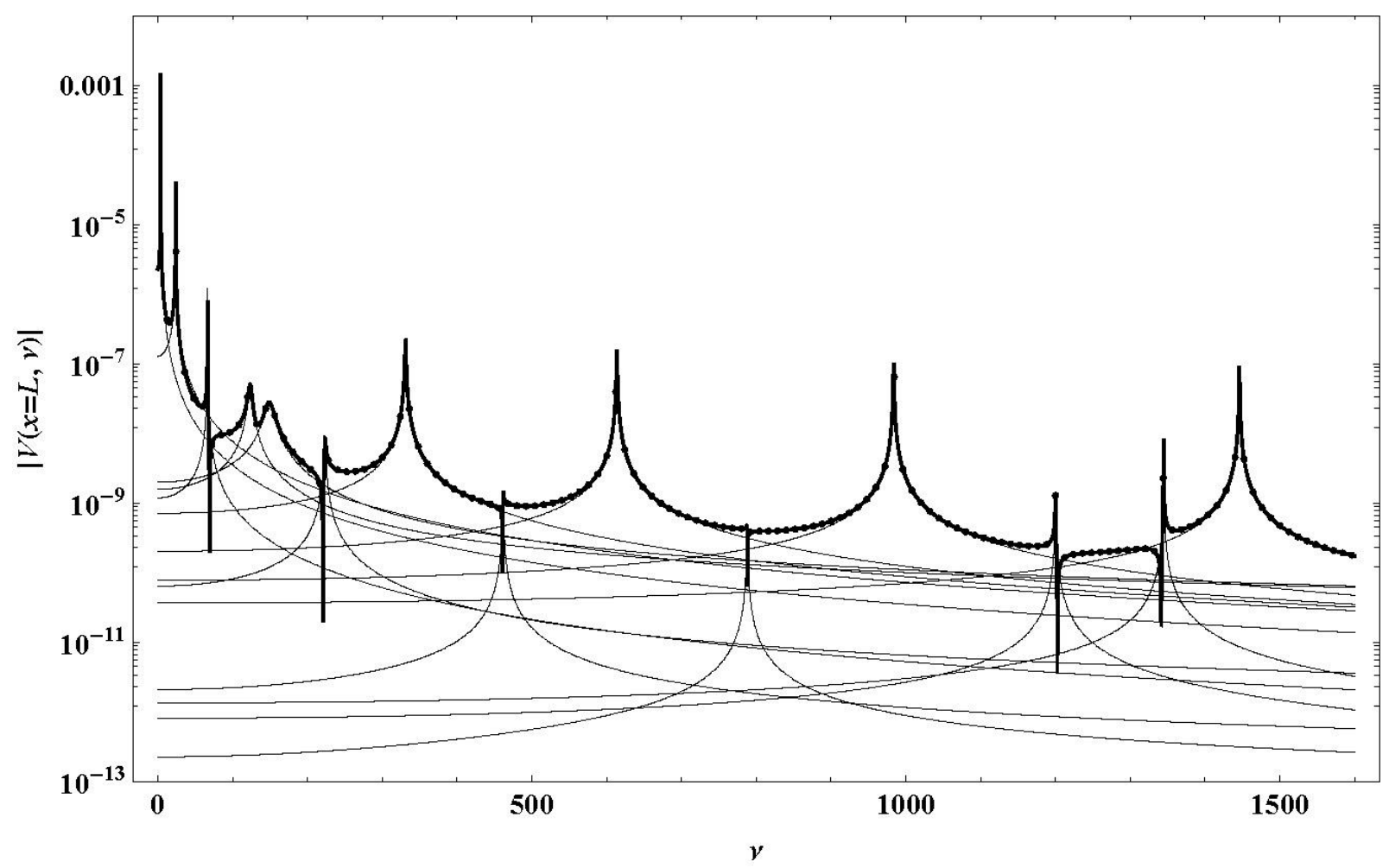

Fig. 3 Frequency response amplitude for the beam deflection at the tip, for $c_{1}=1000 \mathrm{Nm}^{-1} \mathrm{~s}$ and a unit harmonic concentrated load at midspan, as obtained by Eq.(14) (thick black continuous line) and Eq.(27) with $n=15$ modes (black dotted line); single modal contributions (25) (thin black continuous line) 


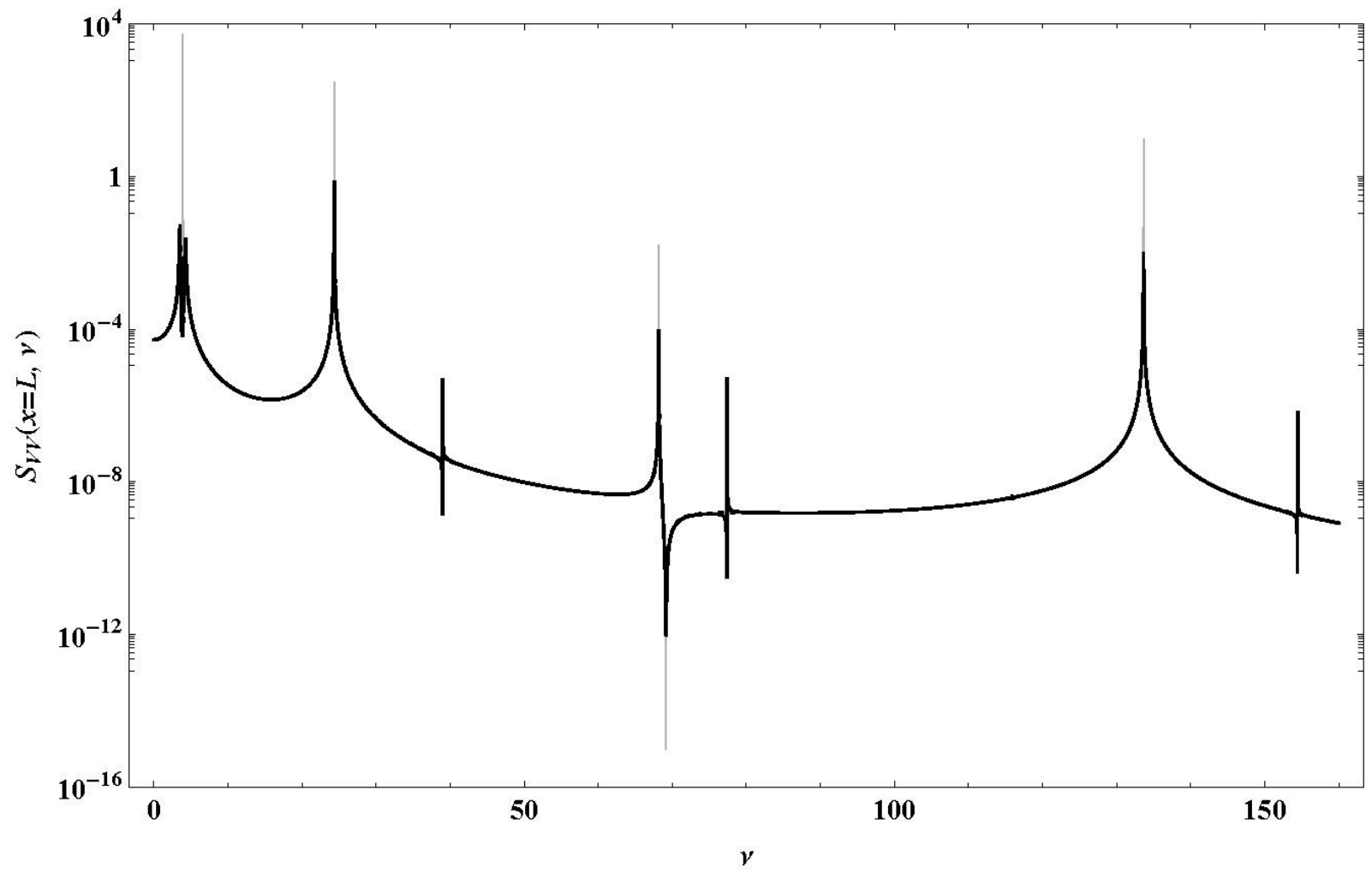

Fig. 4 Tuning frequency $=1$ st natural frequency, PSD (29) of the beam deflection at the tip for $c_{1}=10 \mathrm{Nm}^{-1} \mathrm{~s}$ and a white-noise concentrated load at midspan: with TMD considering (black continuous line) and neglecting (black dashed line) spring inertia effects; without TMD (gray continuous line)

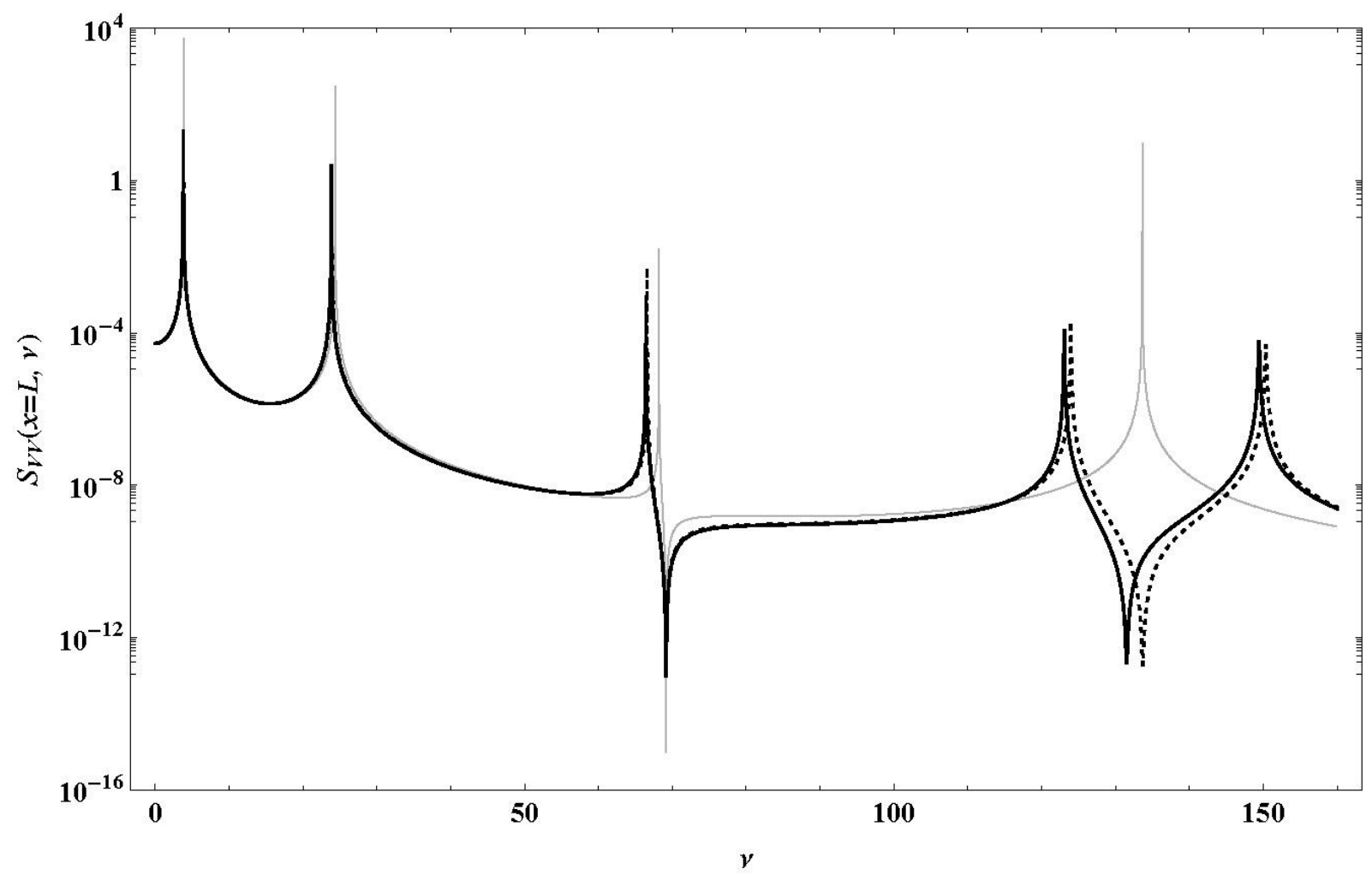

Fig. 5 Tuning frequency $=4$ th natural frequency, PSD (29) of the beam deflection at the tip for $c_{1}=10 \mathrm{Nm}^{-1} \mathrm{~s}$ and a white-noise concentrated load at midspan: with TMD considering (black continuous line) and neglecting (black dashed line) spring inertia effects, without TMD (gray continuous line) 


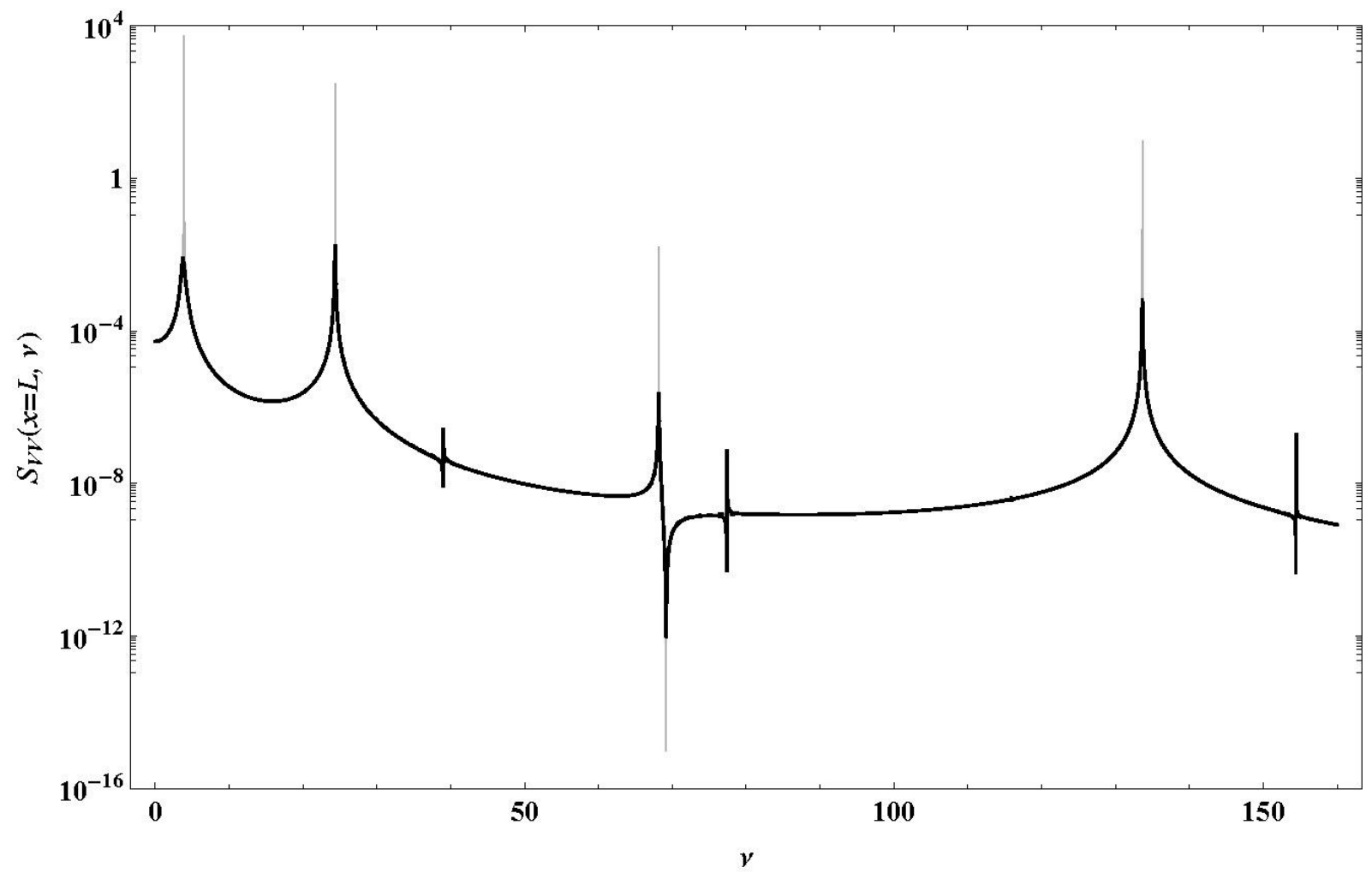

Fig. 6 Tuning frequency $=1$ st natural frequency, PSD (29) of the beam deflection at the tip for $c_{1}=100 \mathrm{Nm}^{-1} \mathrm{~s}$ and a white-noise concentrated load at midspan: with TMD considering (black continuous line) and neglecting (black dashed line) spring inertia effects, without TMD (gray continuous line)

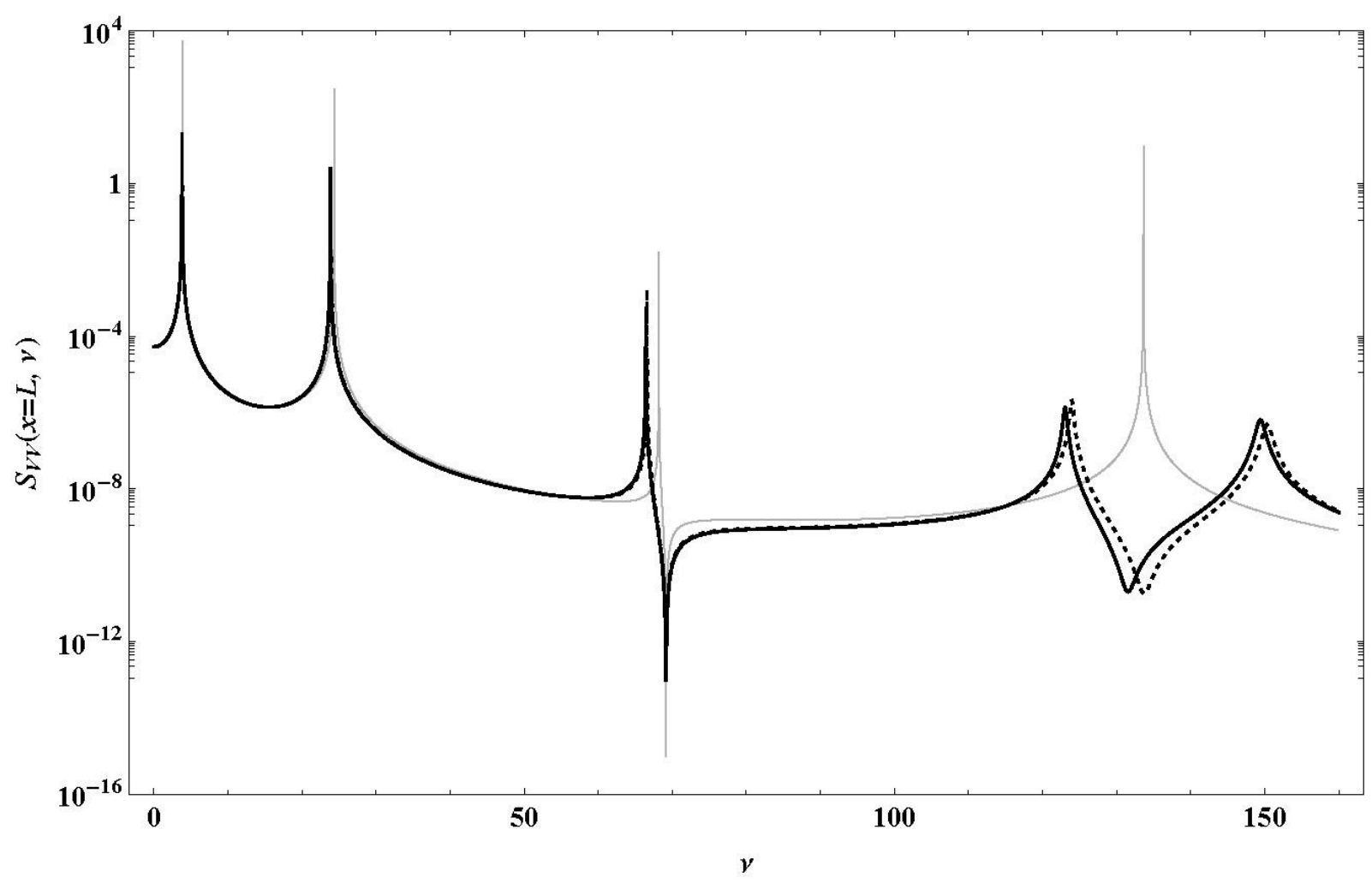

Fig. 7 Tuning frequency $=4$ th natural frequency, PSD (29) of the beam deflection at the tip for $c_{1}=100 \mathrm{Nm}^{-1} \mathrm{~s}$ and a white-noise concentrated load at midspan: with TMD considering (black continuous line) and neglecting (black dashed line) spring inertia effects, without TMD (gray continuous line) 


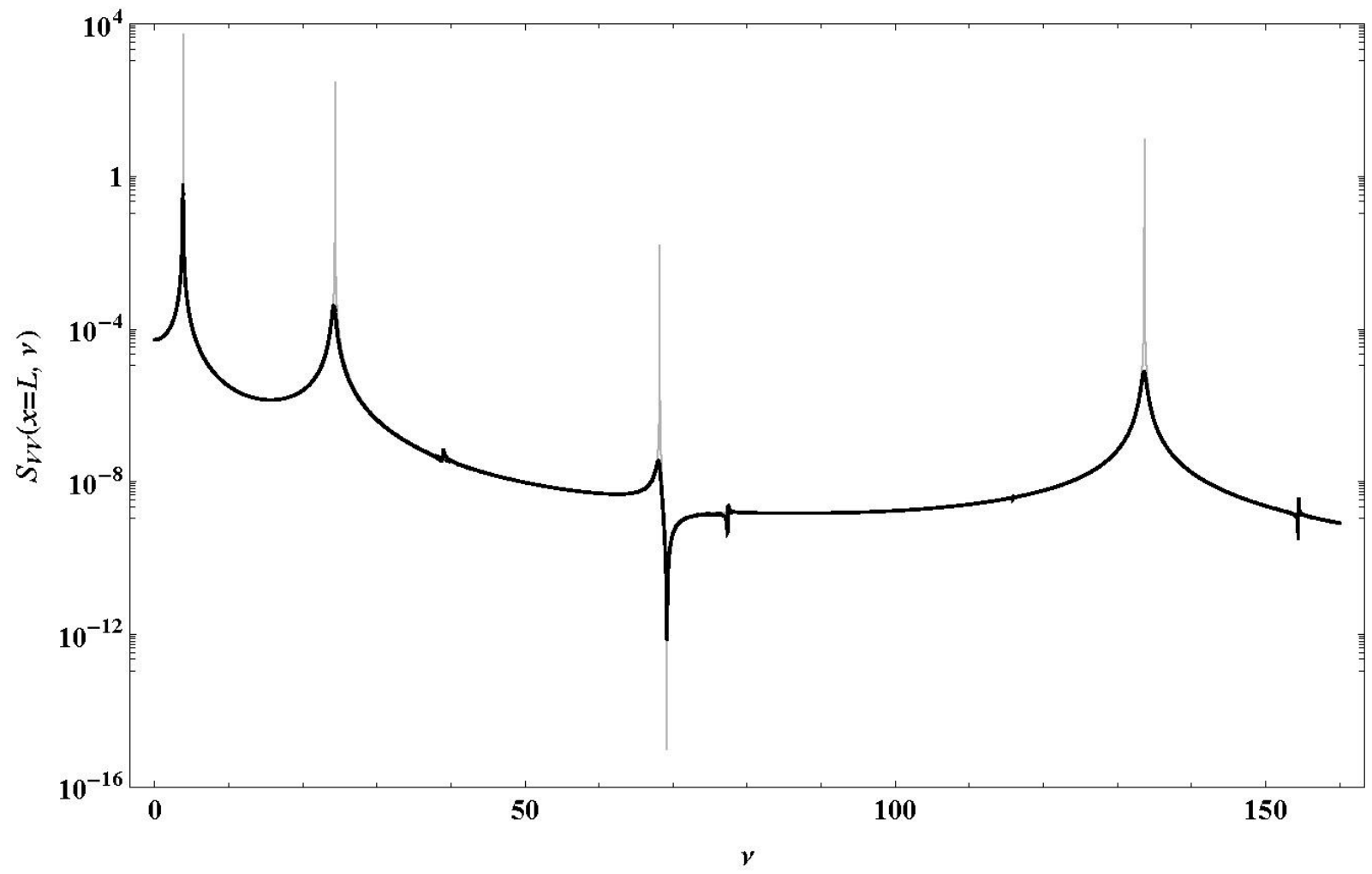

Fig. 8 Tuning frequency $=1$ st natural frequency, PSD (29) of the beam deflection at the tip for $c_{1}=1000 \mathrm{Nm}^{-1} \mathrm{~s}$ and a white-noise concentrated load at midspan: with TMD considering (black continuous line) and neglecting (black dashed line) spring inertia effects, without TMD (gray continuous line)

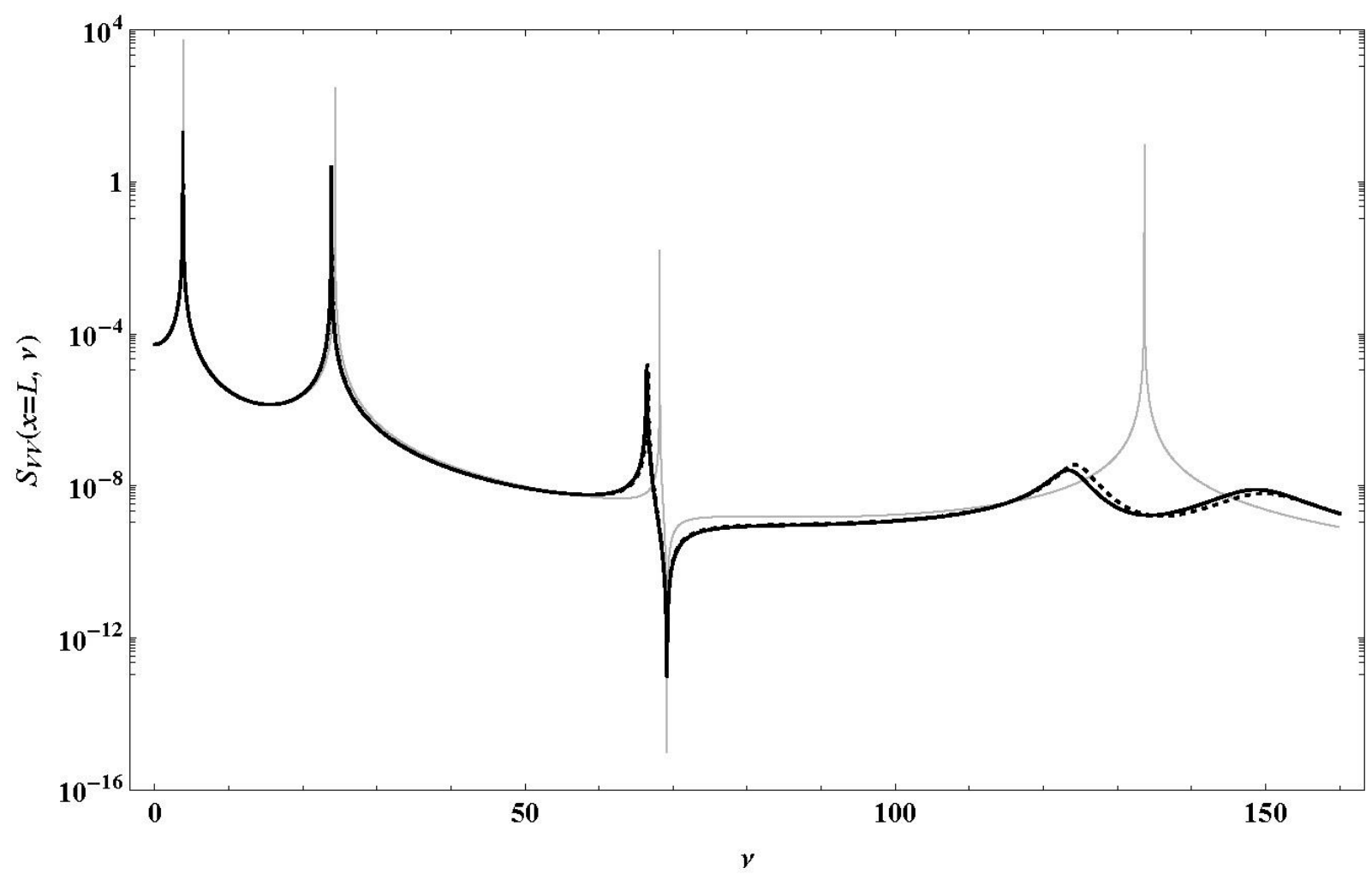

Fig. 9 Tuning frequency $=4$ th natural frequency, PSD (29) of the beam deflection at the tip for $c_{1}=1000 \mathrm{Nm}^{-1} \mathrm{~s}$ and a white-noise concentrated load at midspan: with TMD considering (black continuous line) and neglecting (black dashed line) spring inertia effects, without TMD (gray continuous line) 


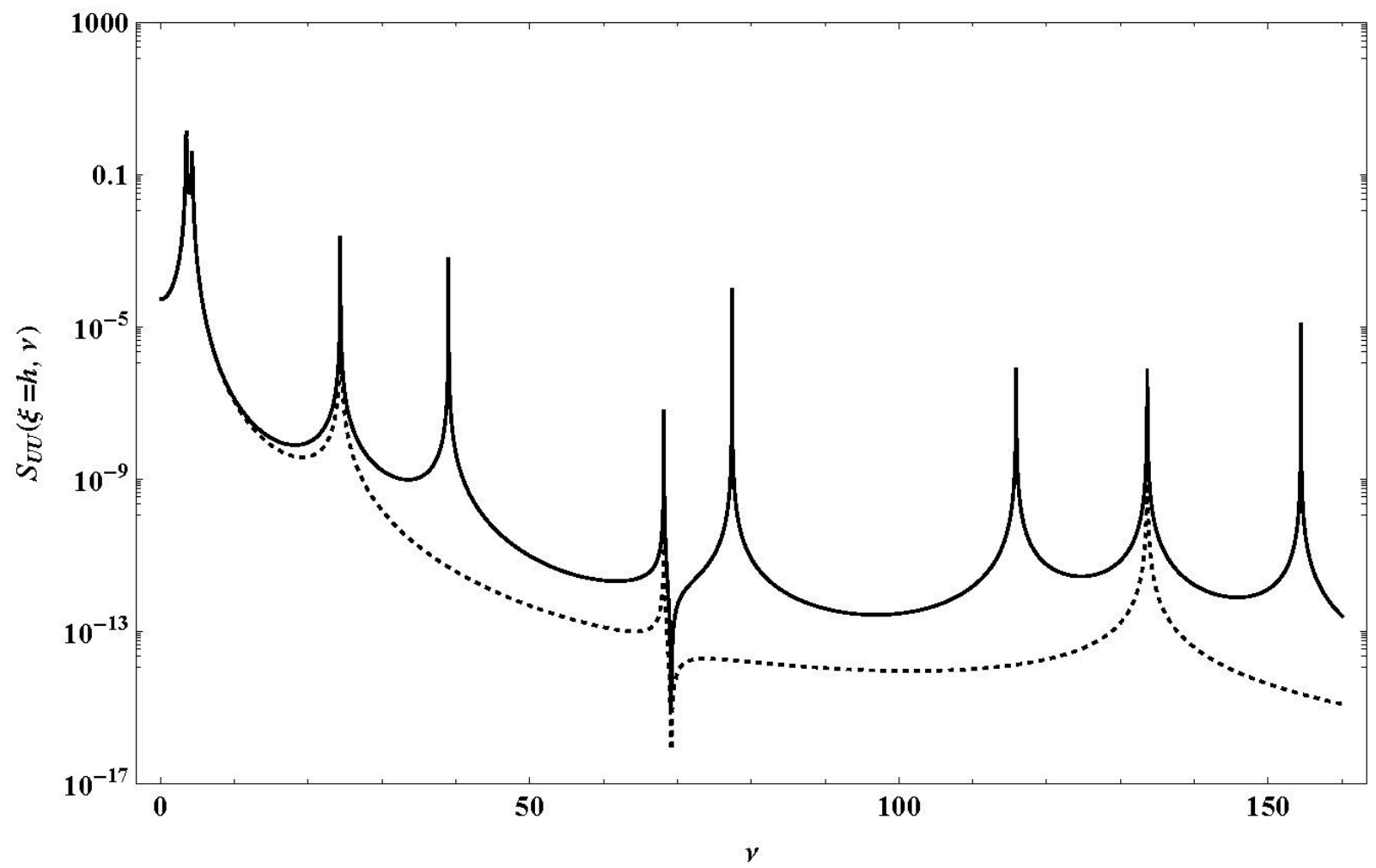

Fig. 10 Tuning frequency $=1$ st natural frequency, PSD (29) of the displacement of the lumped mass $M_{1}$ for $c_{1}=10 \mathrm{Nm}^{-1} \mathrm{~s}$ and a white-noise concentrated load at midspan, considering (black continuous line) and neglecting (black dashed line) spring inertia effects

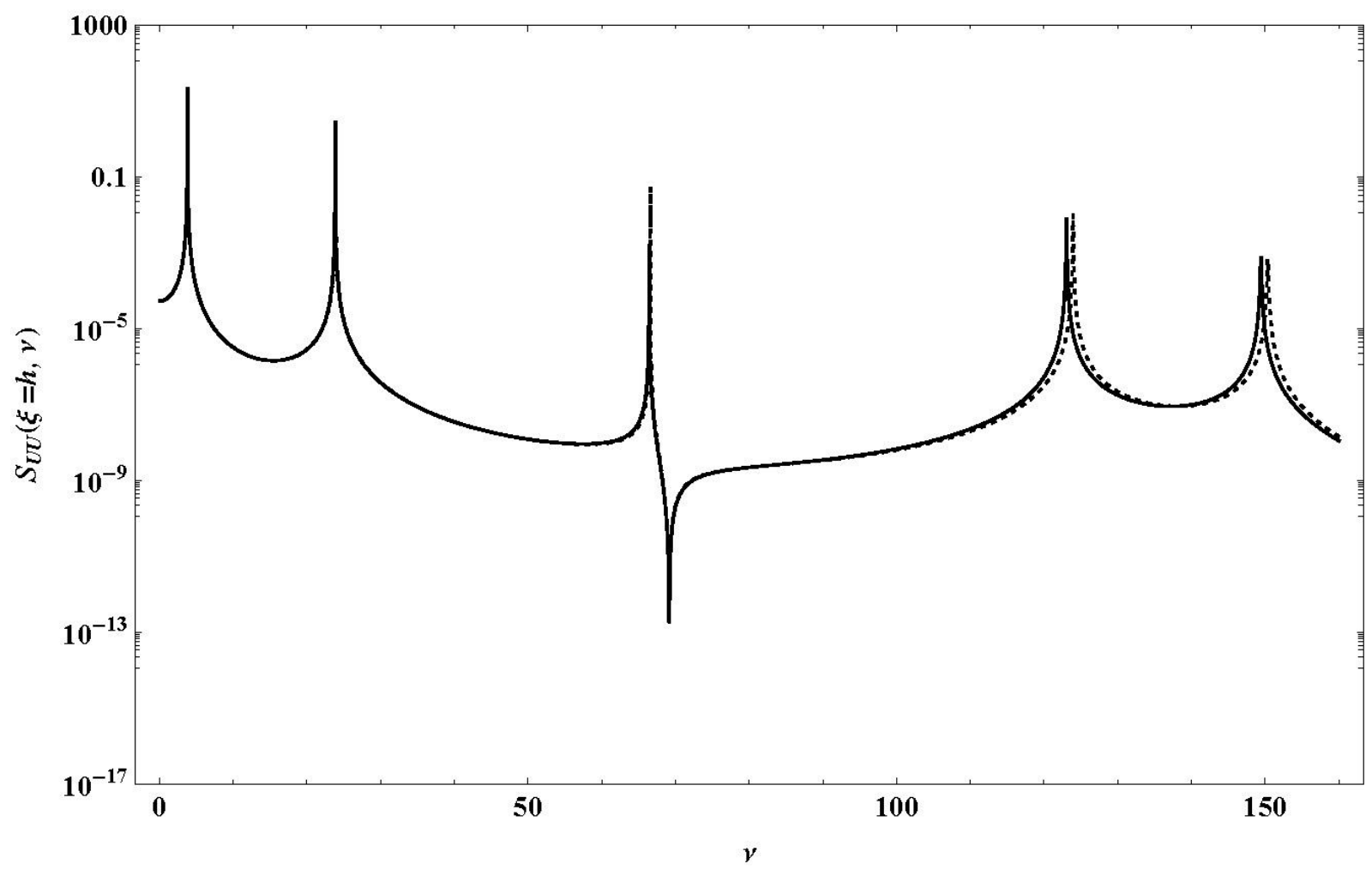

Fig. 11 Tuning frequency $=4$ th natural frequency, PSD (29) of the displacement of the lumped mass $M_{1}$ for $c_{1}=10 \mathrm{Nm}^{-1} \mathrm{~s}$ and a white-noise concentrated load at midspan, considering (black continuous line) and neglecting (black dashed line) spring inertia effects 


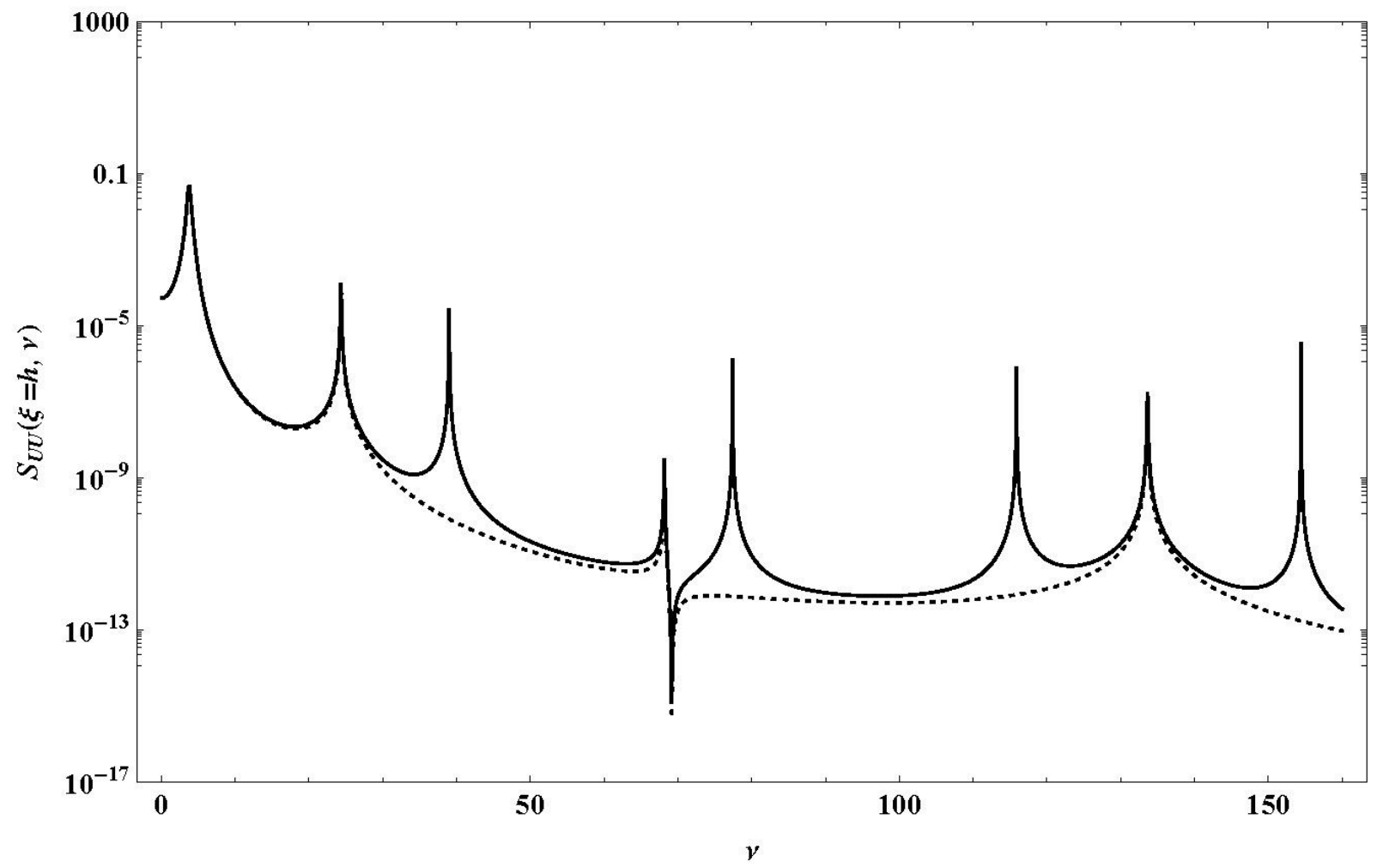

Fig. 12 Tuning frequency $=1$ st natural frequency, PSD (29) of the displacement of the lumped mass $M_{1}$ for $c_{1}=100 \mathrm{Nm}^{-1} \mathrm{~s}$ and a white-noise concentrated load at midspan, considering (black continuous line) and neglecting (black dashed line) spring inertia effects

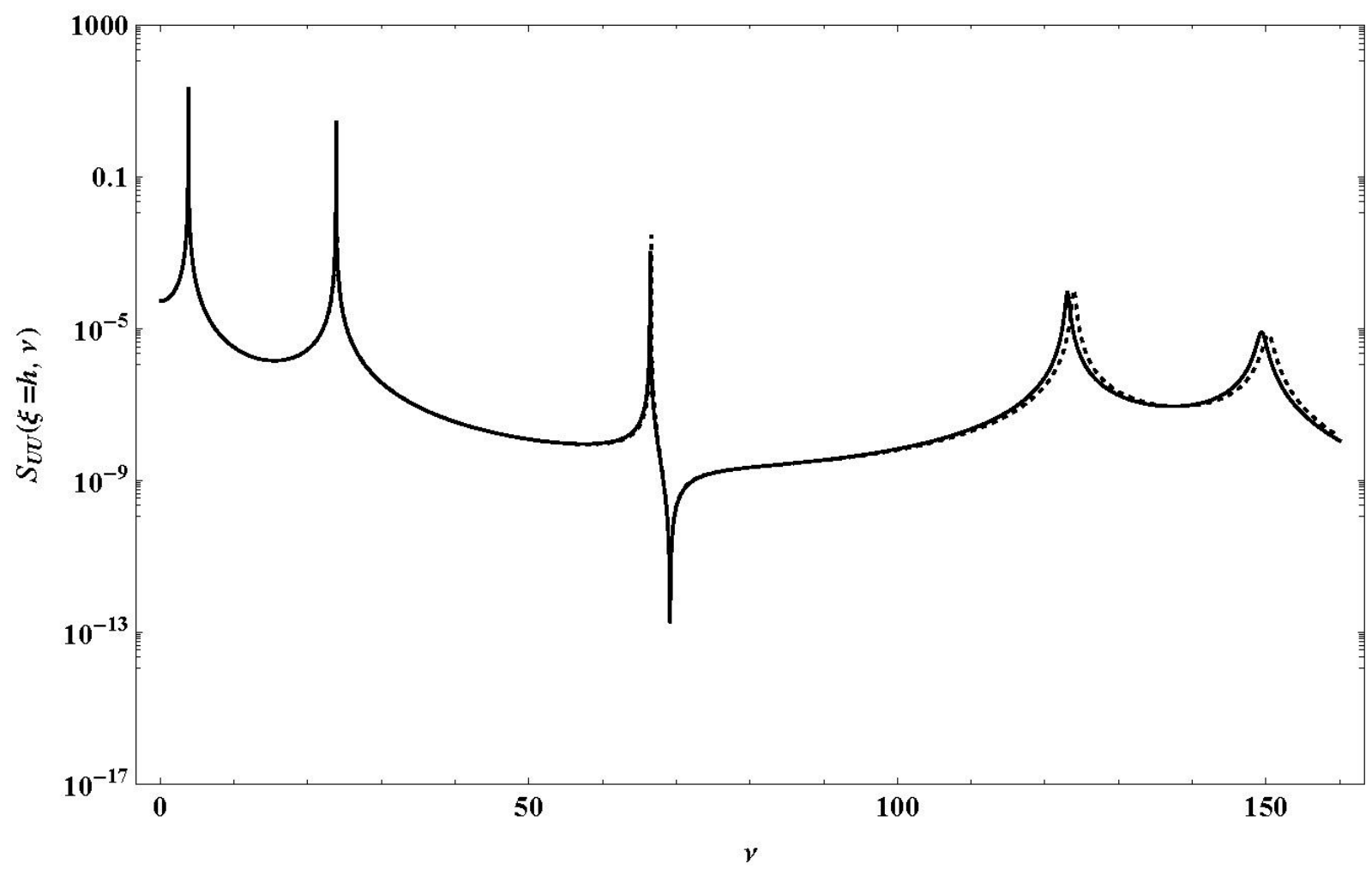

Fig. 13 Tuning frequency $=4$ th natural frequency, PSD (29) of the displacement of the lumped mass $M_{1}$ for $c_{1}=100 \mathrm{Nm}^{-1} \mathrm{~s}$ and a white-noise concentrated load at midspan, considering (black continuous line) and neglecting (black dashed line) spring inertia effects 


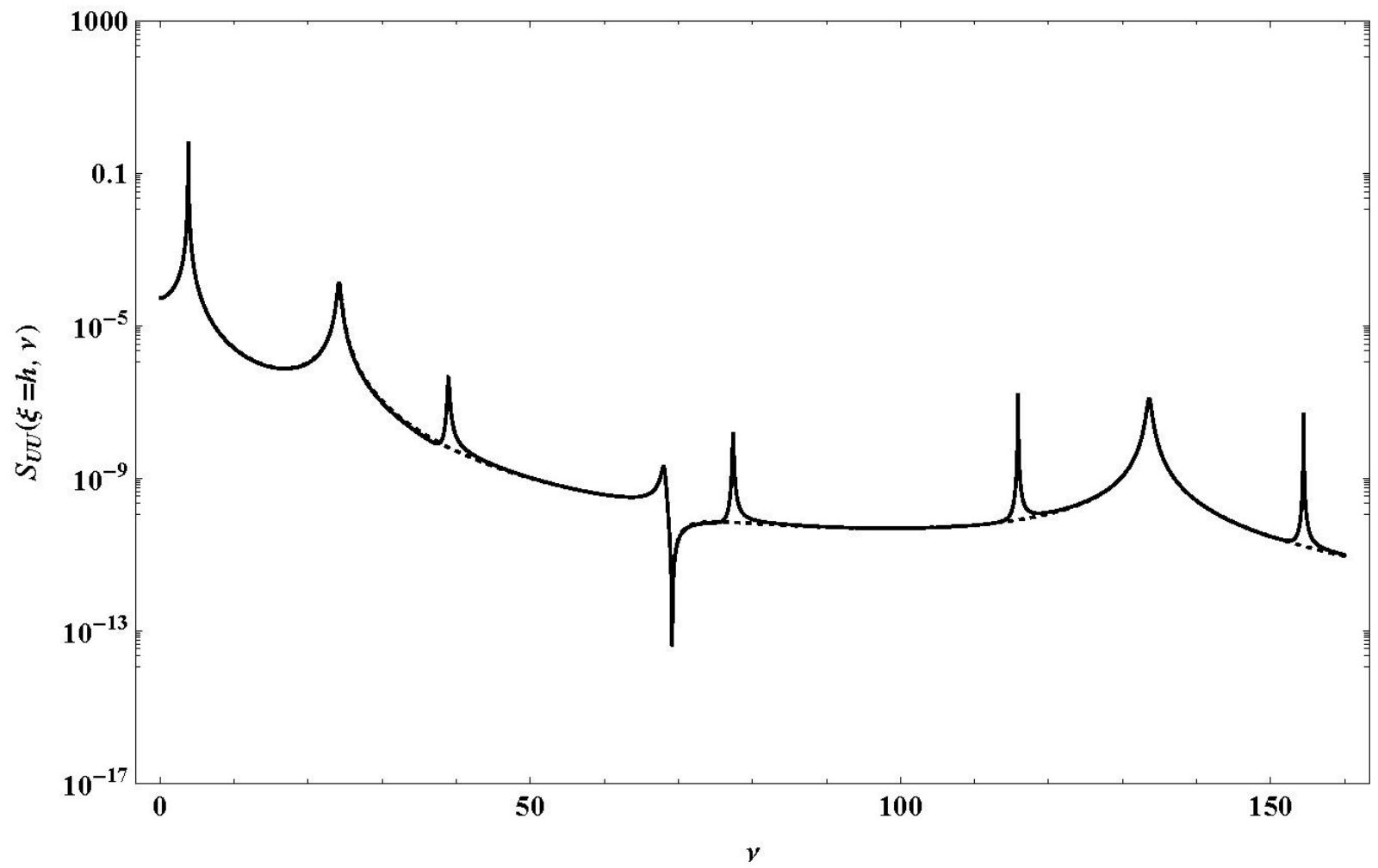

Fig. 14 Tuning frequency = 1st natural frequency, PSD (29) of the displacement of the lumped mass $M_{1}$ for $c_{1}=1000 \mathrm{Nm}^{-1} \mathrm{~s}$ and a white-noise concentrated load at midspan, considering (black continuous line) and neglecting (black dashed line) spring inertia effects

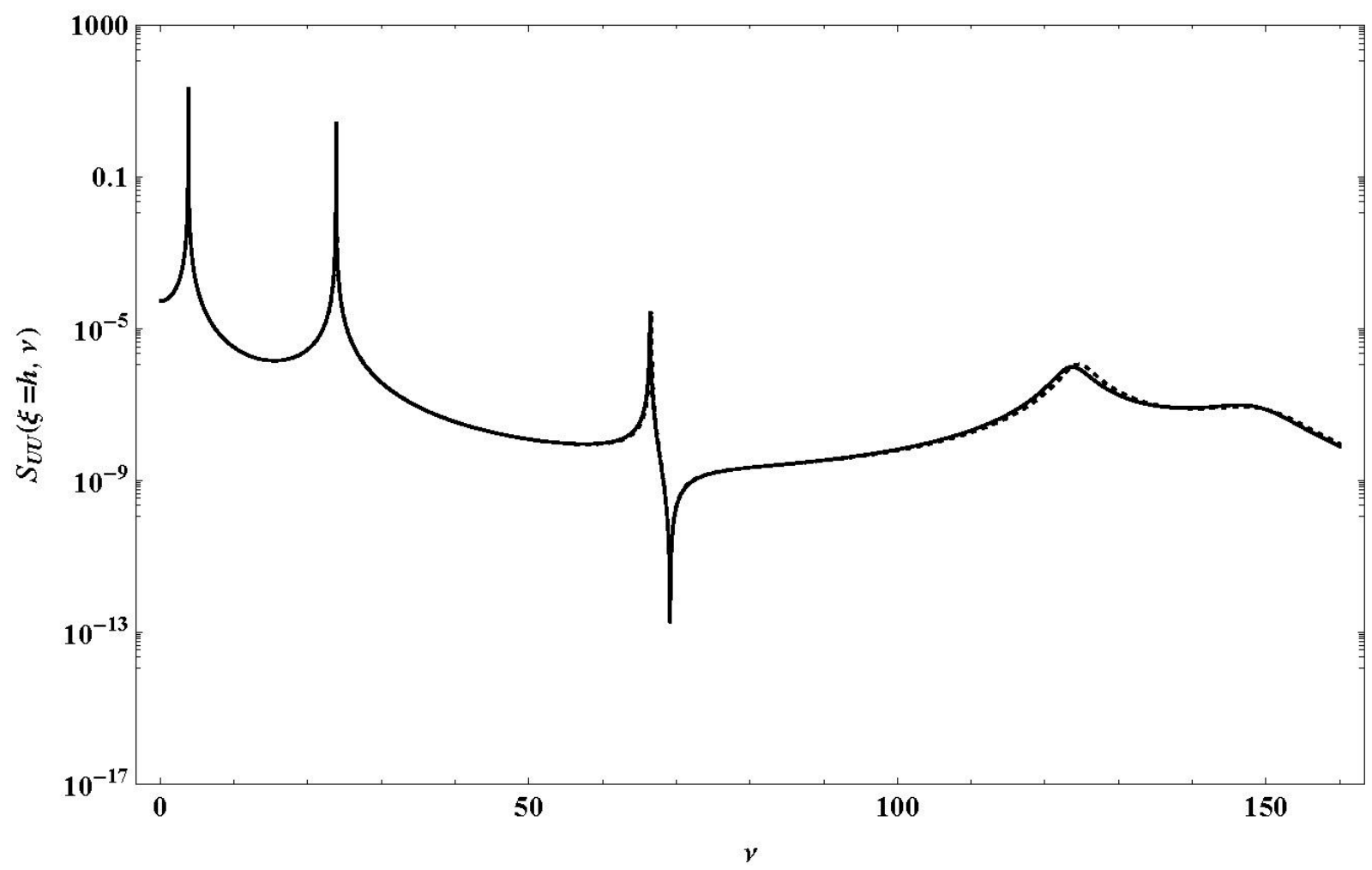

Fig. 15 Tuning frequency $=4$ th natural frequency, PSD (29) of the displacement of the lumped mass $M_{1}$ for $c_{1}=1000 \mathrm{Nm}^{-1} \mathrm{~s}$ and a white-noise concentrated load at midspan, considering (black continuous line) and neglecting (black dashed line) spring inertia effects 


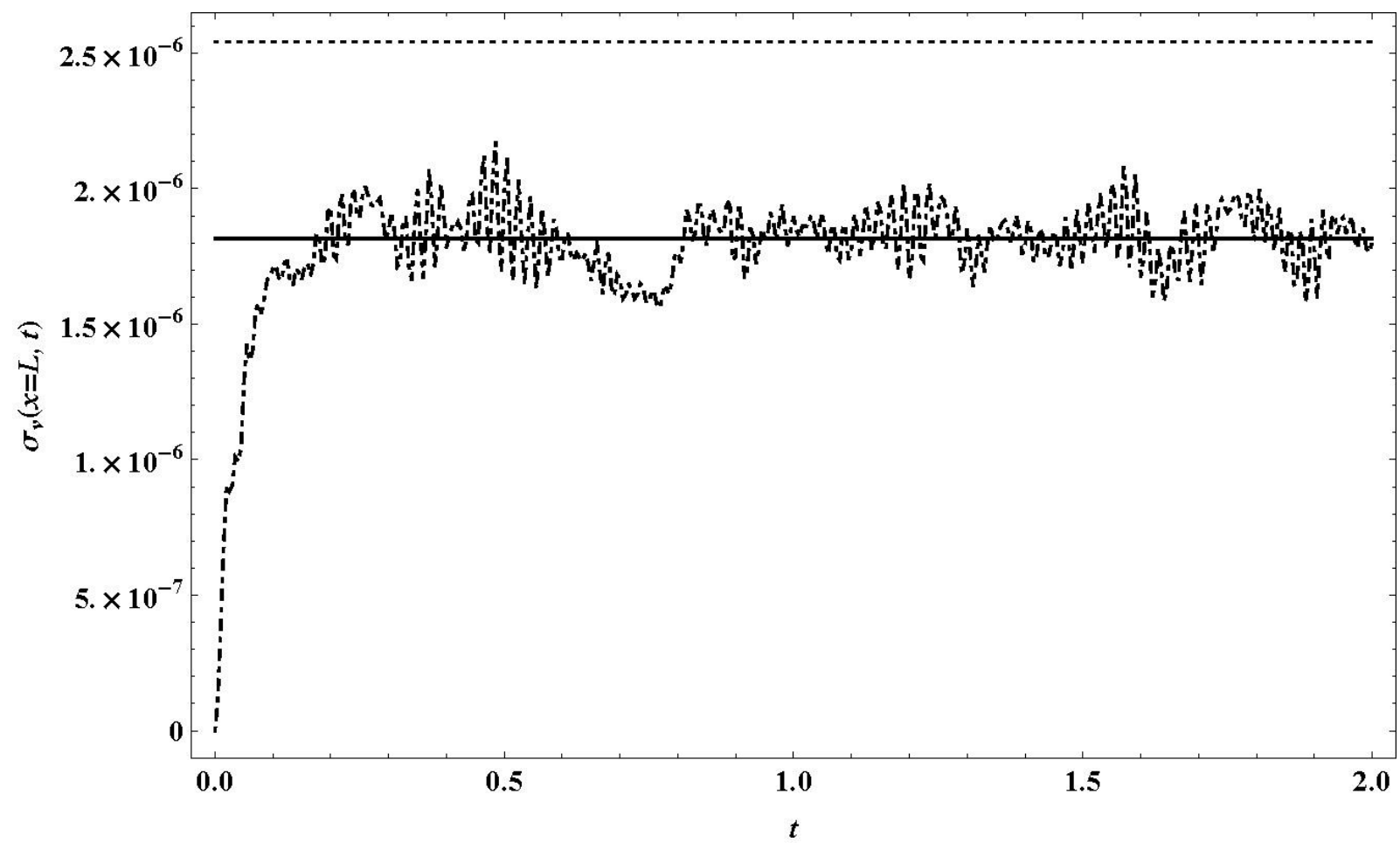

Fig. 16 Tuning frequency $=4$ th natural frequency, variance of the beam deflection at the tip for a colourednoise concentrated load at midspan, with PSD defined in the range $120<v<135$ : considering spring inertia effects, using 5000 Monte Carlo samples (30) (black dot-dashed line) and integrating the PSD (29) (black continuous line); neglecting spring inertia effects, integrating the PSD (29) (gray dashed line)

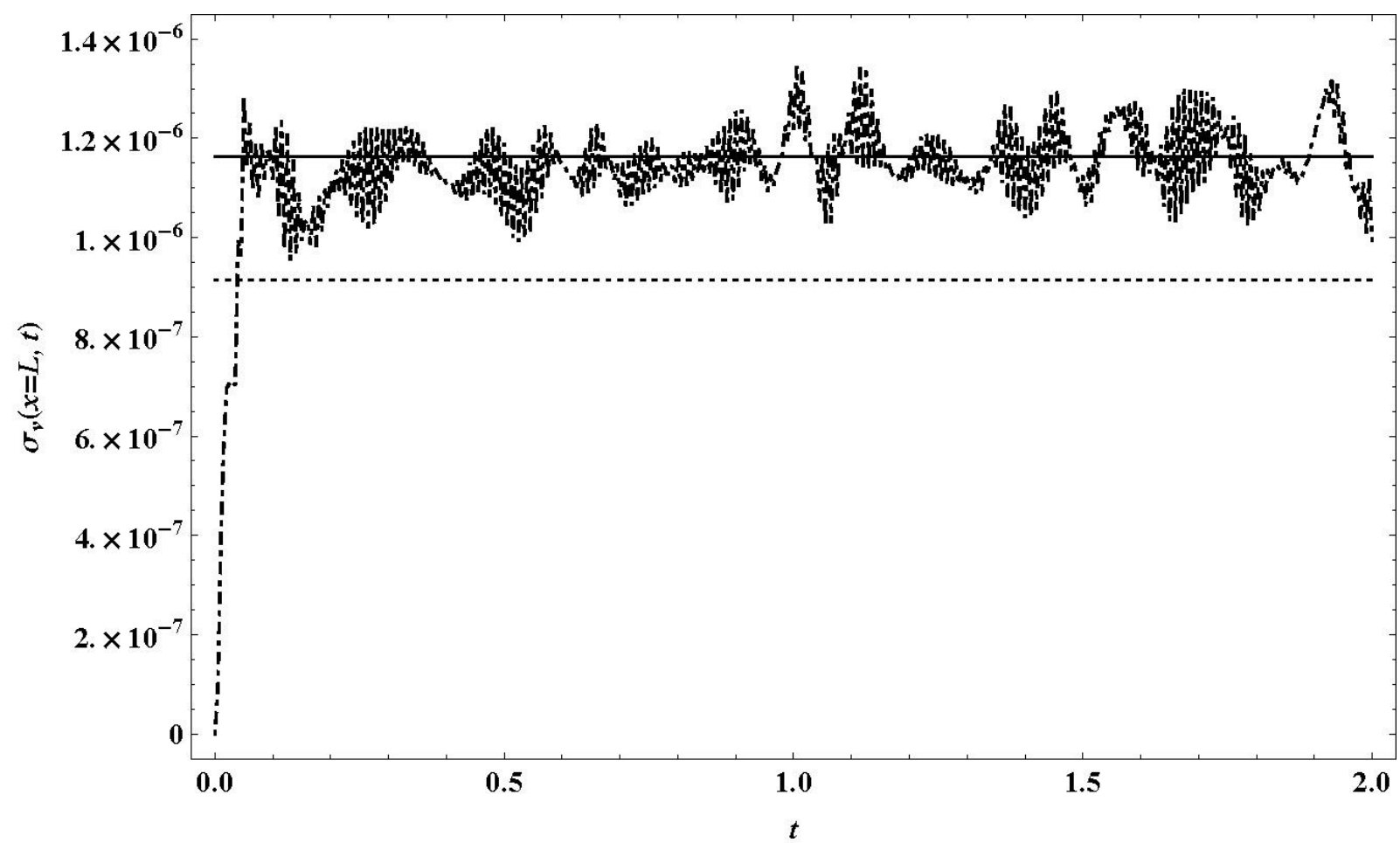

Fig 17 Tuning frequency = 4th natural frequency, variance of the beam deflection at the tip for a colourednoise concentrated load at midspan, with PSD defined in the range $145<v<160$ : considering spring inertia effects, using 5000 Monte Carlo samples (30) (black dot-dashed line) and integrating the PSD (29) (black continuous line); neglecting spring inertia effects, integrating the PSD (29) (gray dashed line) 


\section{CONCLUSIONS}

A comprehensive framework has been presented to calculate the stochastic response of a beam equipped with TMDs where spring inertia effects are considered. The key of the proposed approach is treating the TMD with spring inertia effects as an external support with a pertinent equivalent stiffness. On this basis, the response to stationary and non-stationary loads is built by closed analytical response functions in both frequency and time domains, using the theory of generalized functions. Solutions hold for any number of TMDs along the beam.

For a case study of engineering interest, numerical results have shown that spring inertia effects may play a considerable role in the dynamics of the system and affect the response of the beam to a great extent. This is particularly true at high-tuning frequency, as may be the case in many mechanical applications where high-frequency TMDs are frequently involved [41].

A most suitable generalization of the present work on mass effects in vibration absorbers may concern tuned inerter dampers (TID) [42]. A TID is a device applying a force that linearly depends on the relative acceleration between the device terminals and its inertance, which can be appropriately tuned offering the potential for much higher mass ratios than those obtainable by standard TMDs. Applications of TIDs to structures are gaining increasing attention [43-45]. The stochastic response of beams equipped with multiple TIDs and the effects of inertance will be addressed in a future work, based on the generalized function approach presented in this paper.

\section{APPENDIX A}

Following ref. [30], matrix $\mathbf{W}(x, \omega)$ in Eq.(14) is given as ( $\omega$-dependence is omitted for brevity):

$$
\begin{aligned}
\mathbf{W}(x) & =\boldsymbol{\Omega}(x)+\sum_{j=1}^{N} \mathbf{J}\left(x, x_{j}\right) \boldsymbol{\Phi}_{\boldsymbol{\Omega}}\left(x_{j}\right)+ \\
& \sum_{2 \leq q \leq j} \mathbf{J}\left(x, x_{j}\right) \sum_{(j, m, n, \ldots, r, s) \in \mathbb{N}_{q}^{(j)}} \boldsymbol{\Phi}_{\mathbf{J}}\left(x_{j}, x_{m}\right) \boldsymbol{\Phi}_{\mathbf{J}}\left(x_{m}, x_{n}\right) \cdots \boldsymbol{\Phi}_{\mathbf{J}}\left(x_{r}, x_{s}\right) \boldsymbol{\Phi}_{\mathbf{J}}\left(x_{s}\right)
\end{aligned}
$$

where $\mathbb{N}_{q}^{(j)}=\{(\underbrace{j, m, n, \ldots r, s}_{q}): j>m>n>\ldots>r>s ; m, n, \ldots r, s=1,2, \ldots,(j-1)\}$ is the set including all possible $q$-ples of indexes $(\underbrace{j, m, n, \ldots r, s}_{q})$ such that $j>m>n>\ldots>r>s$, being $2 \leq q \leq j$.

In Eq.(A.1), $\mathbf{J}\left(x, x_{j}\right)$ is the $4 \times 1$ vector 


$$
\mathbf{J}\left(x, x_{j}\right)=\left\{\begin{array}{l}
J_{V, P}\left(x, x_{j}\right) \\
J_{\Theta, P}\left(x, x_{j}\right) \\
J_{M, P}\left(x, x_{j}\right) \\
J_{S, P}\left(x, x_{j}\right)
\end{array}\right\}
$$

while symbol $\boldsymbol{\Phi}_{\mathbf{J}}\left(x_{j}, x_{k}\right)$ denotes the following scalar function:

$$
\boldsymbol{\Phi}\left(x_{j}, x_{k}\right)=-\kappa_{j}(\omega)\left(\mathbf{J}\left(x_{j}, x_{k}\right)\right)_{1}
$$

where notation $(\cdot)_{l}$ indicates the $l^{\text {th }}$ row of the matrix within parenthesis. All terms of $\mathbf{J}\left(x, x_{j}\right)$ can readily be derived from the beam equations

$$
\begin{gathered}
\frac{\overline{\mathrm{d}} S(x)}{\mathrm{d} x}+\sum_{j=1}^{N} R_{j} \delta\left(x-x_{j}\right)+\rho \omega^{2} V(x)=0 \quad \frac{\overline{\mathrm{d}} M(x)}{\mathrm{d} x}=S(x) \\
\frac{\overline{\mathrm{d}} \Theta(x)}{\mathrm{d} x}=-\frac{M(x)}{E I} \quad \frac{\overline{\mathrm{d}} V(x)}{\mathrm{d} x}=\Theta(x)
\end{gathered}
$$

starting from the following particular integrals for deflection associated with a unit force at arbitrary $x=x_{0}$ :

$$
J_{V, P}\left(x, x_{0}\right)=\alpha\left(\sinh \left(\beta\left(x-x_{0}\right)\right)-\sin \left(\beta\left(x-x_{0}\right)\right)\right) H\left(x-x_{0}\right)
$$

where $\alpha=\alpha(\omega)=2^{-1}(E I)^{-1 / 4} \rho^{-3 / 4} \omega^{-3 / 2}$ and $\beta=\beta(\omega)=(E I)^{-1 / 4} \rho^{1 / 4} \omega^{1 / 2}$.

Further, in Eq.(A.1) $\boldsymbol{\Omega}(x)$ depends on the solutions to the homogeneous differential equation associated with Eq.(2), i.e. 


$$
\boldsymbol{\Omega}(x)=\left[\begin{array}{cccc}
\mathrm{e}^{-\beta x} & \mathrm{e}^{\beta x} & \cos (\beta x) & \sin (\beta x) \\
-\beta \mathrm{e}^{-\beta x} & \beta \mathrm{e}^{\beta x} & -\beta \sin (\beta x) & \beta \cos (\beta x) \\
-E I \beta^{2} \mathrm{e}^{-\beta x} & -E I \beta^{2} \mathrm{e}^{\beta x} & E I \beta^{2} \cos (\beta x) & E I \beta^{2} \sin (\beta x) \\
E I \beta^{3} \mathrm{e}^{-\beta x} & -E I \beta^{3} \mathrm{e}^{\beta x} & -E I \beta^{3} \sin (\beta x) & E I \beta^{3} \cos (\beta x)
\end{array}\right]
$$

while $\boldsymbol{\Phi}_{\boldsymbol{\Omega}}\left(x_{j}\right)$ in Eq.(A.1) is the following $1 \times 4$ vector:

$$
\left.\boldsymbol{\Phi}_{\mathbf{\Omega}}\left(x_{j}\right)=\left\{-\kappa_{j}(\omega)\left(\boldsymbol{\Omega}\left(x_{j}\right)\right)\right)_{1}\right\}
$$

Terms in matrices $\boldsymbol{\Phi}_{\Omega}\left(x_{j}\right)$ are derived from the well-known solution to the homogeneous differential equation associated with Eq.(2), using Eqs.(A.4)-(A.5) without Dirac's deltas.

Eq.(A.1) is the basis to derive the analytical expression (14) for the frequency response of the beam, upon using Eq.(15) to calculate the vector of integration constants $\mathbf{d}$ as $\mathbf{d}=\mathbf{B}^{-1} \mathbf{r}$, which is available in a closed form.

Interestingly, from Eq.(14) and specifically the deflection $V(x)$, the exact frequency response can be obtained also in every TMD. For this purpose, notice that using Eqs.(8) for the integration constants $A_{j 1}$ and $A_{j 2}$, axial displacement and force along the rod of every TMD can be expressed as functions of the deflection $V\left(x_{j}\right)$ of the attachment point, in the following form:

$$
\begin{aligned}
& U_{j}(\xi, \omega)=\frac{\beta_{j} \cos \left(\alpha_{j} \omega\left(h_{j}-\xi\right)\right)-\left[\left(M_{j} \omega-\mathrm{i} c_{j}\right) \sin \left(\alpha_{j} \omega\left(h_{j}-\xi\right)\right)-\mathrm{i} c_{j} \sin \left(\alpha_{j} \omega h_{j}\right)\right]}{\beta_{j} \cos \left(\alpha_{j} \omega h_{j}\right)-\left(M_{j} \omega-\mathrm{i} c_{j}\right) \sin \left(\alpha_{j} \omega h_{j}\right)} V\left(x_{j}\right) \\
& N_{j}(\xi, \omega)=E A_{j} \frac{\mathrm{d} U_{j}(\xi, \omega)}{\mathrm{d} \xi}= \\
& \frac{\beta_{j} \omega\left[\left(M_{j} \omega-\mathrm{i} c_{j}\right) \cos \left(\alpha_{j} \omega\left(h_{j}-\xi\right)\right)+\mathrm{i} c_{j} \cos \left(\alpha_{j} \omega h_{j}\right)\right]+\beta_{j}^{2} \omega \sin \left(\alpha_{j} \omega\left(h_{j}-\xi\right)\right)}{\beta_{j} \cos \left(\alpha_{j} \omega h_{j}\right)-\left(M_{j} \omega-\mathrm{i} c_{j}\right) \sin \left(\alpha_{j} \omega h_{j}\right)} V\left(x_{j}\right)
\end{aligned}
$$


Eqs.(A.9) provide analytical expressions for vector $\mathbf{Z}_{j}(\xi, \omega)=\mathbf{Z}_{j}=\left\{\begin{array}{ll}U_{j} & N_{j}\end{array}\right\}^{T}$, i.e. the response in every TMD.

\section{APPENDIX B}

This Appendix summarizes the key steps that lead to derive the closed analytical expression (16) of the impulse response function.

The starting point are the following orthogonality conditions for the deflection modes $V_{k}, V_{n}$, derived following the approach in ref. $[9,32]$

$$
\left(\omega_{k}^{2}-\omega_{n}^{2}\right) \sum_{i=1}^{N} \rho \int_{0}^{L} V_{k} V_{n} \mathrm{~d} x+Q\left(\omega_{k}, \omega_{n}\right)=0
$$

where $Q\left(\omega_{k}, \omega_{n}\right)$ is given as

$$
Q=\sum_{j=1}^{N} V_{k}\left(x_{j}\right)\left(\kappa_{j}\left(\omega_{n}\right)-\kappa_{j}\left(\omega_{k}\right)\right) V_{n}\left(x_{j}\right)
$$

and

$$
\left(\omega_{k}-\omega_{n}\right) \int_{0}^{L} E I \frac{\overline{\mathrm{d}}^{2} V_{k}}{\mathrm{~d} x^{2}} \frac{\overline{\mathrm{d}}^{2} V_{n}}{\mathrm{~d} x^{2}} \mathrm{~d} x+\omega_{k} \omega_{n}\left(\omega_{k}-\omega_{n}\right) \rho \int_{0}^{L} V_{k} V_{n} \mathrm{~d} x+P\left(\omega_{k}, \omega_{n}\right)=0
$$

where $P\left(\omega_{k}, \omega_{n}\right)$ is given as

$$
P=\sum_{j=1}^{N} V_{k}\left(x_{j}\right)\left(\omega_{k} \kappa_{j}\left(\omega_{n}\right)-\omega_{n} \kappa_{j}\left(\omega_{k}\right)\right) V_{n}\left(x_{j}\right)
$$

Under an impulsive loading $p(x, t)=p(x) \delta(t)$, be the impulse response function of the beam given as Eq.(16) of main text, with $g_{n}(t)=\tilde{g}_{n} \mathrm{e}^{\mathrm{i} \omega_{n} t}$ as in Eq.(17). Using the procedure in ref. [9,32], the following equation can be obtained in $\ddot{g}_{n}(t)$ : 


$$
\begin{array}{r}
\sum_{n=1}^{\infty} \frac{\ddot{g}_{n}}{\omega_{n}^{2}} \cdot\left(\sum_{j=1}^{N} \frac{\omega_{n}}{\left(\omega_{k}-\omega_{n}\right)} V_{k}\left(x_{j}\right)\left(\kappa_{j}\left(\omega_{n}\right)-\kappa_{j}\left(\omega_{k}\right)\right) V_{n}\left(x_{j}\right)+\right. \\
\left.+\rho\left(\omega_{k} \omega_{n}+\omega_{n}^{2}\right) \int_{0}^{L} V_{k} V_{n} \mathrm{~d} x\right)=\eta_{k} \delta(t)
\end{array}
$$

where $\ddot{g}_{n}(t)=-\omega_{n}^{2} g_{n}(t)$ being $g_{n}(t)=\tilde{g}_{n} \mathrm{e}^{\mathrm{i} \omega_{n} t}$

Next, consider the expression within parenthesis in Eq.(B.5), which multiplies $\ddot{g}_{n} / \omega_{n}^{2}$ for $n=1, \ldots \infty$. In view of the orthogonality condition (B.1), the expression is equal to zero for $\omega_{n} \neq \omega_{k}$. On the other hand, for $\omega_{n}=\omega_{k}$ the second term within parenthesis in Eq.(B.5) is equal to $2 \rho \omega_{k}^{2} \int_{0}^{L} V_{k} V_{n} \mathrm{~d} x$, while the following limit provides the first term:

$$
\lim _{\omega_{n} \rightarrow \omega_{k}} \frac{\omega_{n} V_{k}\left(x_{j}\right)\left(\kappa_{j}\left(\omega_{n}\right)-\kappa_{j}\left(\omega_{k}\right)\right) V_{n}\left(x_{j}\right)}{\left(\omega_{k}-\omega_{n}\right)}=e_{j}\left(\omega_{k}\right) V_{k}^{2}\left(x_{j}\right)
$$

where

$$
e_{j}\left(\omega_{k}\right)=\omega_{k}^{2} \sum_{l=1}^{4} \frac{r_{j, l}\left(\omega_{k}\right)}{s_{j}\left(\omega_{k}\right)}
$$

In Eq.(B.7),

$$
\begin{gathered}
r_{j, 1}\left(\omega_{k}\right)=\omega_{k}\left[\omega_{k}\left(2 h_{j} \gamma_{j} \omega_{k}-\mathrm{i} \gamma_{j}\right) M_{j}^{2}-4 h_{j} \gamma_{j} c_{j}^{2}-2 c_{j}\left(c_{j}+\mathrm{i} 2 h_{j} \gamma_{j} \omega_{k}\right) M_{j}\right] \\
r_{j, 2}\left(\omega_{k}\right)=E A_{j} \gamma_{j}\left[2 \omega_{k}\left(h_{j} \gamma_{j}+M_{j}\right)-\mathrm{i} c_{j}\right]+4 \gamma_{j} c_{j}\left[h_{j} \omega_{k}\left(c_{j}+\mathrm{i} \omega_{k} M_{j}\right)+\mathrm{i} E A_{j}\right] \cos \left(\alpha_{j} h_{j} \omega_{k}\right) \\
r_{j, 3}\left(\omega_{k}\right)=\left[\omega_{k} c_{j} M_{j}\left(2 c_{j}+\mathrm{i} \omega_{k} M_{j}\right)+E A_{j} \gamma_{j}\left(2 M_{j} \omega_{k}-\mathrm{i} 3 c_{j}\right)\right] \cos \left(2 \alpha_{j} h_{j} \omega_{k}\right) \\
r_{j, 4}\left(\omega_{k}\right)=\mathrm{i} 4 \beta_{j}\left(h_{j} \gamma_{j} \omega_{k}+\mathrm{i} c_{j}\right) c_{j} \sin \left(\alpha_{j} h_{j} \omega_{k}\right) \\
r_{j, 5}\left(\omega_{k}\right)=\beta_{j}\left(E A_{j} \gamma_{j}+2 c_{j}^{2}+\mathrm{i} 4 \omega_{k} c_{j} M_{j}-M_{j}^{2} \omega_{k}^{2}\right) \sin \left(2 \alpha_{j} h_{j} \omega_{k}\right)
\end{gathered}
$$


while $s_{j}\left(\omega_{k}\right)$ is

$$
s_{j}\left(\omega_{k}\right)=2 \omega_{k}\left[\beta_{j} \cos \left(\alpha_{j} h_{j} \omega_{k}\right)+\mathrm{i}\left(c_{j}+\mathrm{i} M_{j} \omega_{k}\right) \sin \left(\alpha_{j} h_{j} \omega_{k}\right)\right]^{2}
$$

Now, integrating Eq.(B.5) finally provides

$$
\dot{g}_{k}\left(0^{+}\right)=\frac{\eta_{k}}{\Pi_{k}}
$$

with $\Pi_{k}$ given as Eq.(20) of the main text. Considering that $\dot{g}_{k}\left(0^{+}\right)=\mathrm{i} \omega g_{k}\left(0^{+}\right)=\mathrm{i} \omega \tilde{g}_{k}$, the expression (18) for the complex coefficient $\tilde{g}_{k}$ is finally derived.

\section{REFERENCES}

[1] Den Hartog JP (1962) Mechanical vibrations. McGraw-Hill, New York.

[2] Housner GW, et al. (1997) Structural control: Past, present and future. J Eng Mech 123(9): 897-971. https://doi.org/10.1061/(ASCE)0733-9399(1997)123:9(897)

[3] Spencer BF, Nagarajaiah S (2003) State of the art of structural control. J Struct Eng 129(7): 845-856. https://doi.org/10.1061/(ASCE)0733-9445(2003)129:7(845)

[4] Elias S, Matsagar V (2017) Research developments in vibration control of structures using passive tuned mass dampers. Аnnu Rev Control 44: 129-156. https://doi.org/10.1016/j.arcontrol.2017.09.015

[5] Chen Y-H, Huang Y-H (2004) Timoshenko beam with tuned mass dampers and its design curves. J Sound Vib 278(4-5): 873-888. https://doi.org/10.1016/j.jsv.2003.10.013

[6] Yang F, Sedaghati R, Esmailzadeh E (2009) Vibration suppression of non-uniform curved beams under random loading using optimal tuned mass damper. J Vib Control 15(2): 233261. https://doi.org/10.1177/1077546308091220

[7] Younesian D, Esmailzadeh E, Sedaghati R (2006) Passive vibration control of beams subjected to random excitations with peaked PSD. J Vib Control 12(9): 941-953. https://doi.org/10.1177/1077546306068060

[8] Das AK, Dey SS (1992) Effects of tuned mass dampers on random response of bridges. Comp Struct 43(4): 745-750. https://doi.org/10.1016/0045-7949(92)90518-5 
[9] Adam C, Di Lorenzo S, Failla G, Pirrotta A (2017) On the moving load problem in beam structures equipped with tuned mass dampers. Meccanica 52: 3101-3115. https://doi.org/10.1007/s11012-016-0599-4

[10] Samani FS, Pellicano F (2009) Vibration reduction on beams subjected to moving loads using linear and nonlinear dynamic absorbers. J Sound Vib 325(4-5): 742-754. https://doi.org/10.1016/j.jsv.2009.04.011

[11] Lee C-L, Chen Y-T, Chung L-L, Wang Y-P (2006) Optimal design theories and applications of tuned mass dampers. Eng Struct 28(1), 43-53. https://doi.org/10.1016/j.engstruct.2005.06.023

[12] Salvi J, Rizzi E, Rustighi E, Ferguson NS (2018) Optimum tuning of passive tuned mass dampers for the mitigation of pulse-like responses. J Vib Acoust 140(6): 061014. https://doi.org/10.1115/1.4040475

[13] Salvi J, E Rizzi E (2017) Optimum earthquake-tuned TMDs: Seismic performance and new design concept of balance of split effective modal masses. Soil Dyn Earthq Eng 101: 67-80. https://doi.org/10.1016/j.soildyn.2017.05.029

[14] Rayleigh JWS (1945) The theory of sound. Vol. 1, 2nd ed., Dover, New York.

[15] Yamamoto Y (1999) Spring's effective mass in spring mass system free vibration. J Sound Vib 220(3): 564-570. https://doi.org/10.1006/jsvi.1998.1944

[16] Wu J-S, Hsu T-F (2007) Free vibration analyses of simply supported beams carrying multiple point masses and spring-mass systems with mass of each helical spring considered. Int $J$ Mech Sci 49: 834-852. https://doi.org/10.1016/j.ijmecsci.2006.11.015

[17] Cha PD, Chan M, Nielsen G (2008) Eigenfrequencies of an arbitrarily supported beam carrying multiple in-span elastic rod-mass systems. J Vib Acoust 130: 061008. https://doi.org/10.1115/1.2980384

[18] Gürgöze M, Zeren S, Bicak MMA (2008) On the consideration of the masses of helical springs in damped combined systems consisting of two continua. Struct Eng Mech 28(2): 167188. https://doi.org/10.12989/sem.2008.28.2.167.

[19] Gürgöze M, Zeren S (2011) Consideration of the masses of helical springs in forced vibrations of damped combined systems. Mech Res Commun 38: 239-243. https://doi.org/10.1016/j.mechrescom.2011.03.001

[20] Gürgöze M (2005) On the eigenfrequencies of a cantilever beam carrying a tip spring-mass system with mass of the helical spring considered. J Sound Vib 282: 1221-1230. https://doi.org/10.1016/j.jsv.2004.04.020 
[21] Gürgöze M, Çakar O, Zeren S (2006) On the frequency equation of a combined system consisting of a simply supported beam and in-span helical spring-mass with mass of the helical spring considered. $J$ Sound Vib 295: 436-449. https://doi.org/10.1016/j.jsv.2006.01.027

[22] Wu J-J (2006) Use of equivalent mass method for free vibration analyses of a beam carrying multiple two-dof spring-mass systems with inertia effect of the helical springs considered. Int J Numer Meth Eng 65: 653-678. https://doi.org/10.1002/nme.1460

[23] Wu J-J (2006) Study on the inertia effect of helical spring of the absorber on suppressing the dynamic responses of a beam subjected to a moving load. J Sound Vib 297: 981-999. https://doi.org/10.1016/j.jsv.2006.05.011

[24] Yavari A, Sarkani S, Moyer ET (2000) On applications of generalized functions to beam bending problems. Int $J$ Solids Struct 37: 5675-5705. https://doi.org/10.1016/S00207683(99)00271-1

[25] Falsone G (2002) The use of generalised functions in the discontinuous beam bending differential equation. Int J Eng Educ 18(3): 337-343.

[26] Falsone $G$ (2018) The use of generalized functions modeling the concentrated loads on Timoshenko beams. Struct Eng Mech 67(4): 385-390. https://doi.org/10.12989/sem.2018.67.4.385

[27] Caddemi S, Caliò I (2013) The exact explicit dynamic stiffness matrix of multi-cracked Euler-Bernoulli beam and applications to damaged frame structures. J Sound Vib 332(12): 3049-3063. https://doi.org/10.1016/j.jsv.2013.01.003

[28] Caddemi S, Caliò I, Cannizzaro F (2017) The dynamic stiffness matrix (DSM) of axially loaded multi-cracked frames. Mech Res Commun 84: 90-97. https://doi.org/10.1016/j.mechrescom.2017.06.012

[29] Burlon A, Failla G, Arena F (2016) Exact frequency response analysis of axially loaded beams with viscoelastic dampers. Int $J$ Mech Sci 115-116: 370-384. https://doi.org/10.1016/j.ijmecsci.2016.07.024

[30] Failla G (2016) An exact generalised function approach to frequency response analysis of beams and plane frames with the inclusion of viscoelastic damping. J Sound Vib 360: 171202. https://doi.org/10.1016/j.jsv.2015.09.006

[31] Burlon A, Failla G, Arena F (2017) Coupled bending and torsional free vibrations of beams with in-span supports and attached masses. Eur $J$ Mech A/Solids 66: 387-411. https://doi.org/10.1016/j.euromechsol.2017.07.015 
[32] Burlon A, Failla G, Arena F (2018) Exact frequency response of two-node coupled bendingtorsional beam element with attachments. Appl Math Model 63: 508-537. https://doi.org/10.1016/j.apm.2018.06.047

[33] Di Lorenzo S, Adam C, Burlon A, Failla G, Pirrotta A (2018) Flexural vibrations of discontinuous layered elastically bonded beams. Compos Part B: Eng 135: 175-188. https://doi.org/10.1016/j.compositesb.2017.09.059

[34] Failla G (2019) An exact modal analysis approach to vibration analysis of structures with mass-spring subsystems and rotational joints. $J$ Sound Vib 438: 191-219. https://doi.org/10.1016/j.jsv.2018.09.025

[35] Donà M, Palmeri A, Lombardo M, Cicirello A (2015) An efficient two-node finite element formulation of multi-damaged beams including shear deformation and rotatory inertia. Comput Struct 147: 96-106. https://doi.org/10.1016/j.compstruc.2014.10.002

[36] Wang J, Qiao P (2007) Vibration of beams with arbitrary discontinuities and boundary conditions. J Sound Vib 308: 12-27. https://doi.org/10.1016/j.jsv.2007.06.071

[37] Oliveto G, Santini A, Tripodi E (1997) Complex modal analysis of a flexural vibrating beam with viscous end conditions. J Sound Vib 200(3): 327-345. https://doi.org/10.1006/jsvi.1996.0717

[38] Veletsos AS, Ventura CE (1986) Modal analysis of non-classically damped linear systems. Earthq Eng Struct Dyn 14: 217-243. https://doi.org/10.1002/eqe.4290140205

[39] Mathematica (2008). Version 7.0, Wolfram Research Inc., Champaign.

[40] Shinozuka M, Deodatis G (1991) Simulation of stochastic processes by spectral representation. Appl Mech Rev 44(4): 191-204. https://dx.doi.org/10.1115/1.3119501

[41] Vibratec (1988) High frequency - tuned mass damper. Stockholm. http://vibratec.se/products/high-frequency-tuned-mass-damper/

[42] Krenk S, Høgsberg J (2016) Tuned resonant mass or inerter-based absorbers: unified calibration with quasi-dynamic flexibility and inertia correction. Proc. R. Soc. A 472: 20150718. http://dx.doi.org/10.1098/rspa.2015.0718

[43] De Domenico D, Impollonia N, Ricciardi G (2018) Soil-dependent optimum design of a new passive vibration control system combining seismic base isolation with tuned inerter damper. Soil Dyn Earthq Eng 105: 37-53. https://doi.org/10.1016/j.soildyn.2017.11.023

[44] De Domenico D, Ricciardi G (2018) An enhanced base isolation system equipped with optimal tuned mass damper inerter (TMDI). Earthq Eng Struct Dyn 47(5): 1169-1192. https://doi.org/10.1002/eqe.3011 
[45] Shi X, Zhu S (2018) Dynamic characteristics of stay cables with inerter dampers. J Sound Vib 1 423: 287-305. https://doi.org/10.1016/j.jsv.2018.02.042 
$x_{0}$ 
Figure 3

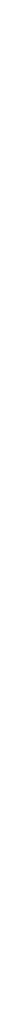

Click here to

access/dow 


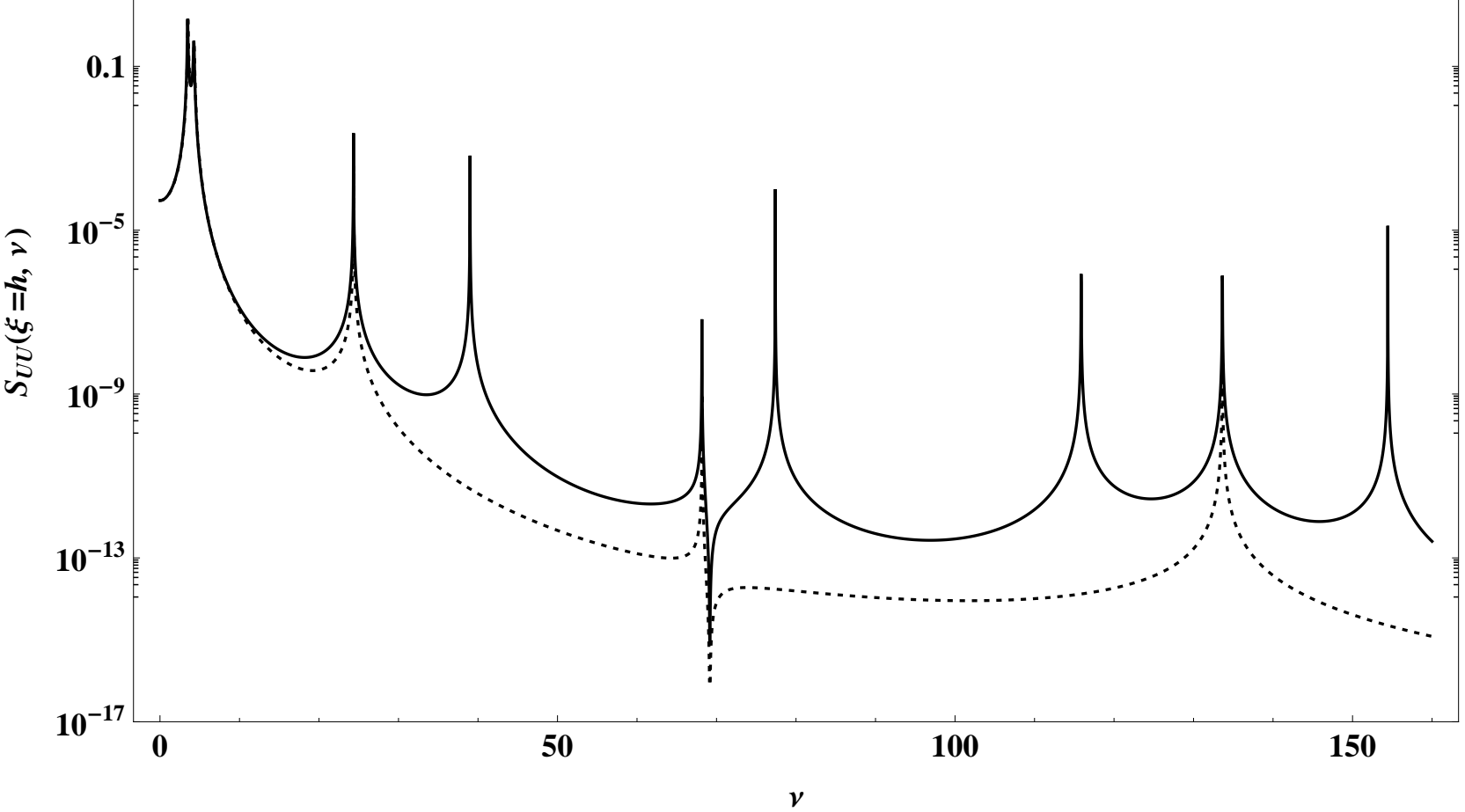




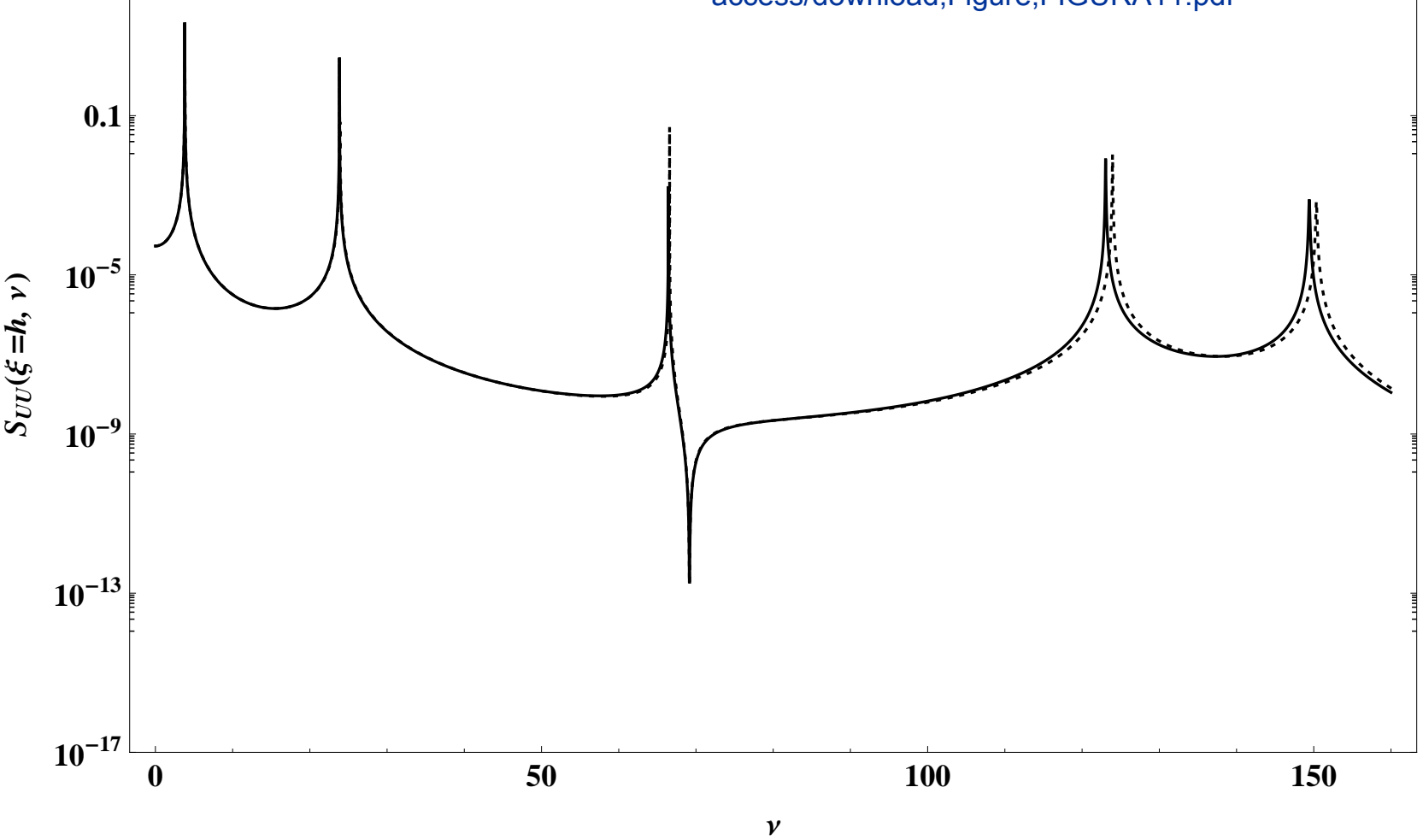




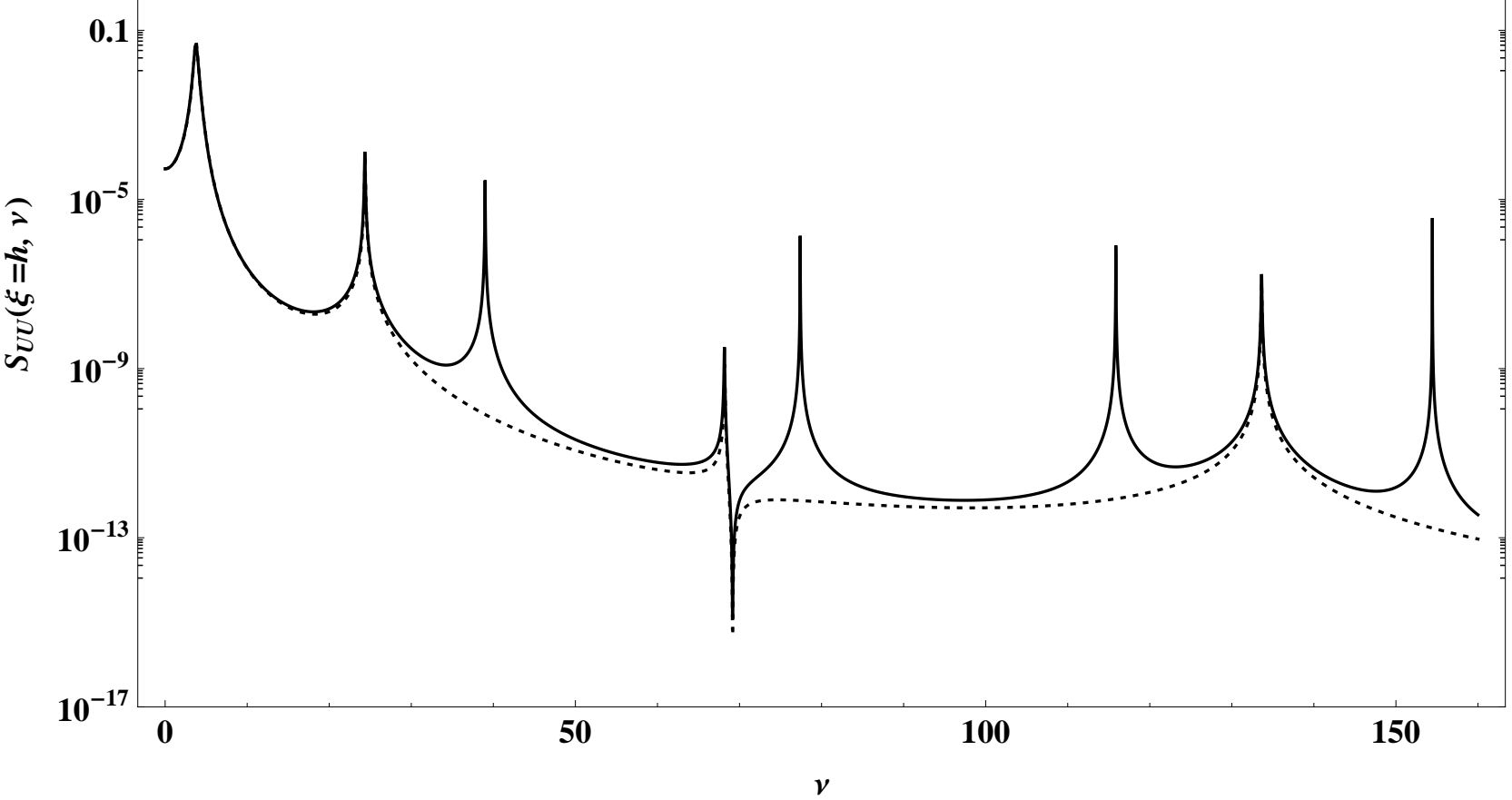




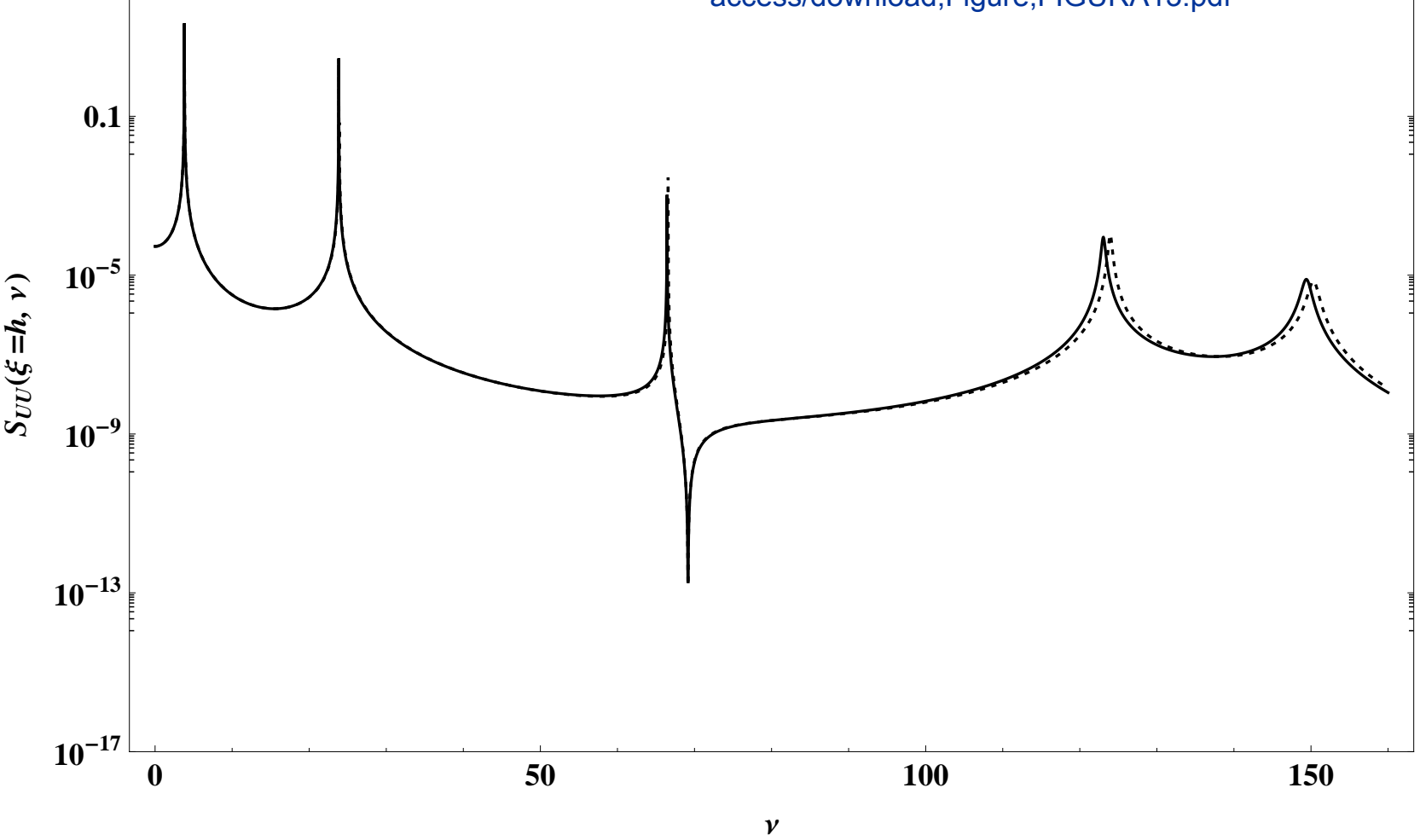




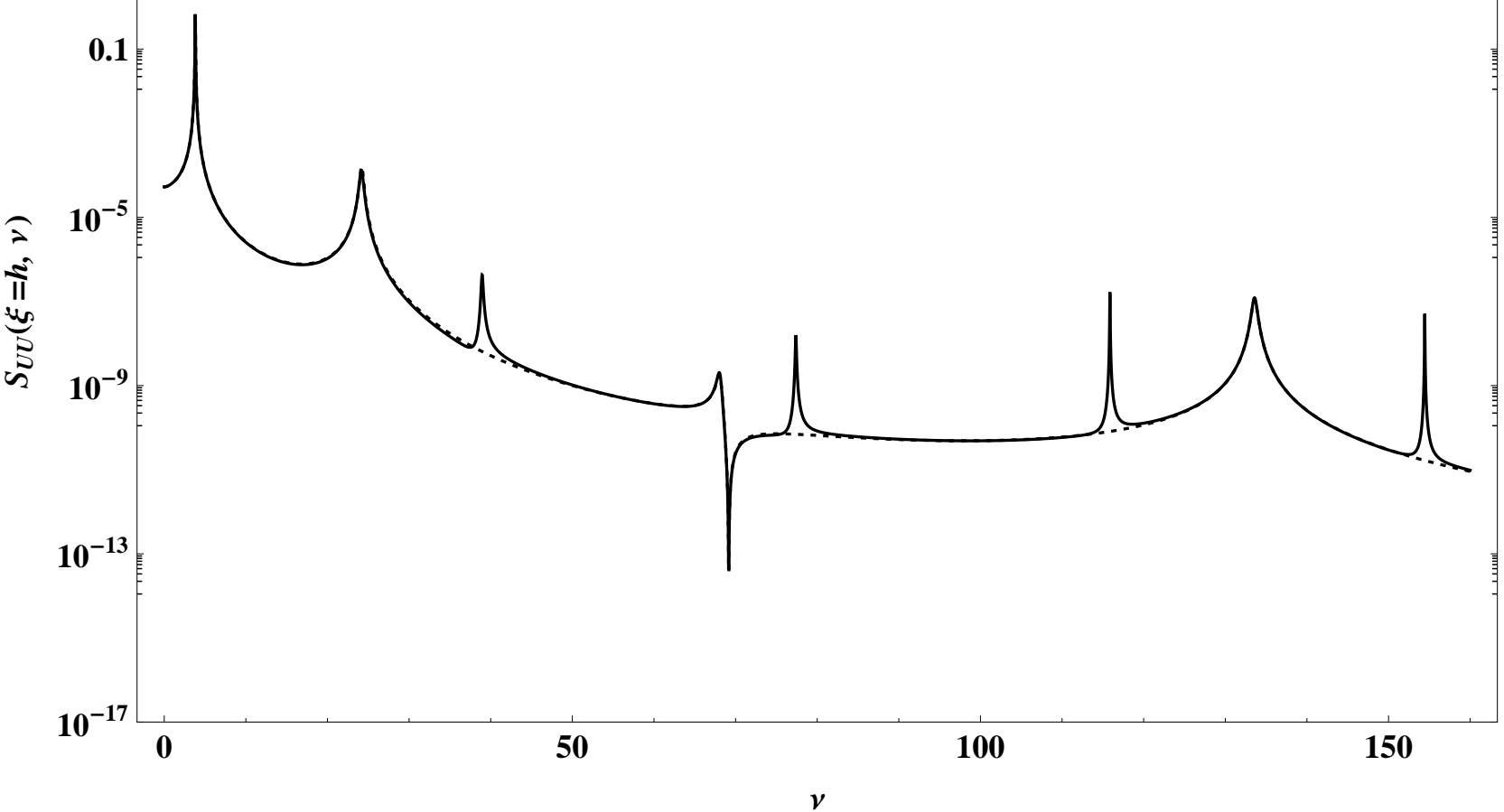




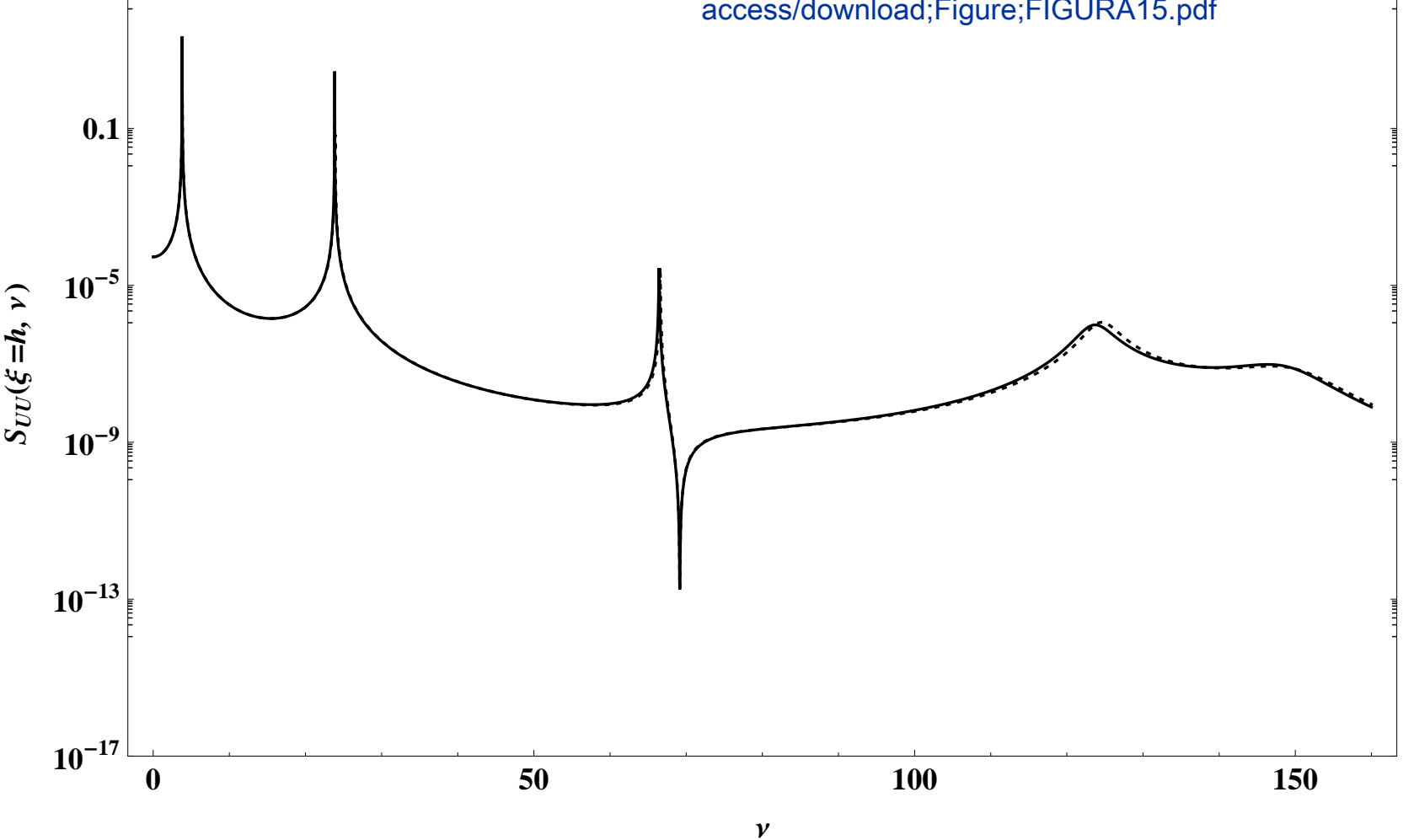


Figute $\times 170^{-6}$

access/download;Figure;FIGURA17.pdf

accessldowioad;Figure;FIGURA17.pdf

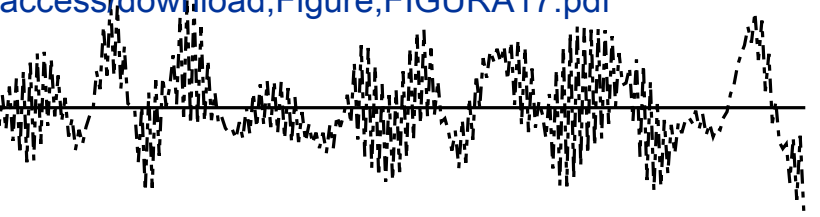

8. $\times 10^{-7}$

6. $\times 10^{-7}$

4. $\times 10^{-7}$

2. $\times 10^{-7}$

$\mathbf{0}$

0.0 